\title{
Platelet PD-L1 reflects collective intratumoral PD-L1 expression and predicts immunotherapy response in non-small cell lung cancer
}

Clemens Hinterleitner 1,2, Jasmin Strähle ${ }^{3}$, Elke Malenke ${ }^{4}$, Martina Hinterleitner (1) 1,2, Melanie Henning 1,2, Marco Seehawer (10 1,2, Tatjana Bilich 2,5,6, Jonas Heitmann²,6, Martina Lutz ${ }^{2,6}$, Sven Mattern7, Sophia Scheuermann (10) ${ }^{2,4}$, Marius Horger ${ }^{8}$, Stefanie Maurer ${ }^{2,6,9}$, Juliane Walz (1) 2,5,6, Falko Fend (1) ${ }^{7}$, Rupert Handgretinger ${ }^{2,4}$, Christian Seitz (D) 2,4 Bettina Weigelin (1) ${ }^{10}$, Stephan Singer ${ }^{7}$, Helmut Salih ${ }^{2,6}$, Oliver Borst ${ }^{11}$, Hans-Georg Kopp ${ }^{12} \&$ Lars Zender (1) 1,2,13凶

Immune-checkpoint inhibitors $(\mathrm{ICI})$ have transformed oncological therapy. Up to $20 \%$ of all non-small cell lung cancers (NSCLCs) show durable responses upon treatment with $\mathrm{ICl}$, however, robust markers to predict therapy response are missing. Here we show that blood platelets interact with lung cancer cells and that PD-L1 protein is transferred from tumor cells to platelets in a fibronectin 1, integrin $\alpha 5 \beta 1$ and GPIb $\alpha$-dependent manner. Platelets from NSCLC patients are found to express PD-L1 and platelet PD-L1 possess the ability to inhibit CD4 and CD8 T-cells. An algorithm is developed to calculate the activation independent adjusted PD-L1 payload of platelets (pPD-L1Adj.), which is found to be superior in predicting the response towards $\mathrm{ICl}$ as compared to standard histological PD-L1 quantification on tumor biopsies. Our data suggest that platelet PD-L1 reflects the collective tumor PD-L1 expression, plays important roles in tumor immune evasion and overcomes limitations of histological quantification of often heterogeneous intratumoral PD-L1 expression.

\footnotetext{
${ }^{1}$ Department of Medical Oncology \& Pneumology (Internal Medicine VIII), University Hospital Tuebingen, Tuebingen, Germany. ${ }^{2}$ DFG Cluster of Excellence 2180 'Image-guided and Functional Instructed Tumor Therapy' (iFIT), University of Tuebingen, Tuebingen, Germany. ${ }^{3}$ Department of Hematology, Oncology and Immunology, University Hospital Tuebingen, Tuebingen, Germany. ${ }^{4}$ Department of Pediatric Hematology and Oncology, University Hospital Tuebingen, Tuebingen, Germany. ${ }^{5}$ Institute for Cell Biology, Department of Immunology, University of Tuebingen, Tuebingen, Germany. ${ }^{6}$ Clinical Collaboration Unit Translational Immunology, German Cancer Consortium (DKTK), Department of Internal Medicine, University Hospital Tuebingen, Tuebingen, Germany. ${ }^{7}$ Department of Pathology and Neuropathology, University Hospital Tuebingen, Tuebingen, Germany. ${ }^{8}$ Department of Radiology, University Hospital Tuebingen, Tuebingen, Germany. ${ }^{9}$ Department of Radiology, Memorial Sloan Kettering Cancer Center, New York, NY, USA. ${ }^{10}$ Werner Siemens Imaging Center, Department of Preclinical Imaging and Radiopharmacy, Eberhard Karls University Tuebingen, Tuebingen, Germany. ${ }^{11}$ University Hospital, Department of Cardiology and Angiology, Eberhard Karls University of Tuebingen, Tuebingen, Germany. ${ }^{12}$ Robert-Bosch-Hospital, Department of Molecular and Pneumological Oncology, Stuttgart, Germany. ${ }^{13}$ German Cancer Research Consortium (DKTK), Partner Site Tübingen, German Cancer Research Center

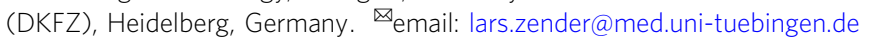


mmune-checkpoint receptors like CTLA4 and PD-1 are crucial for preventing excessive immune responses and autoimmunity ${ }^{1,2}$. Seminal discoveries made by Allison and Honjo provided preclinical proof of concept data that blockage of CTLA4 and PD1 signaling unleashes marked anti-tumor immune responses $^{3,4}$. Clinical evaluation revealed remarkable therapeutic potential of immune-checkpoint inhibition in human cancer patients and for the first time allowed for long term survival of patients with advanced metastasized solid tumors ${ }^{5-9}$. Besides melanoma patients, especially patients suffering from non-small cell lung cancer (NSCLC) benefit from treatment with antibodies inhibiting the PD1 and CTLA4 immune checkpoints ${ }^{5-7}$. Nevertheless, simple and robust biomarkers to predict therapy responses towards ICI are still missing.

With 1.8 million deaths per year, lung cancer represents one of the most frequent and lethal cancers worldwide ${ }^{10}$. In the US 254,170 new lung cancer cases are expected to be diagnosed in $2021^{11}$. Given the high frequency of lung cancer and the cost of checkpoint inhibitory therapies, the lack of robust biomarkers to select patients who best possibly benefit from ICI represents a major burden for our health systems. Histological quantification of intratumoral PD-L1 expression is routinely performed in an attempt to predict therapy responses towards ICI, however, only an insufficient correlation between detection of PD-L1 expression in tumor biopsies and the overall response rate (ORR) was found ${ }^{12}$. In lung cancer, evaluation of smoking history, tumor mutational burden (TMB), microsatellite instability (MSI), high expression of CTLA4, low expression of CX3CL1 and infiltration of $\mathrm{CD} 8+\mathrm{T}$ cells within the tumor microenvironment (TME) seems to be superior in predicting therapy responses towards anti-PD-1/PD-L1 directed ICI ${ }^{13-15}$ when compared to histopathological PD-L1 quantification, however these markers so far could not be translated into a robust and clinically easy to use biomarker signature.

Here, we show that platelets, during their frequent interaction with tumor cells, ingest PD-L1 and present it on their surface, a process which is dependent on fibronectin, a5 31 and GPIba. PDL1 expressing platelets are detected in the TME and peripheral blood of NSCLC patients. The functionality of platelet PD-L1 (pPD-L1) is confirmed by inhibition of CD4+ and CD8+ activity. pPD-L1 correlates with tumor stage/grade and the occurrence of metastases. We develop an algorithm allowing to calculate the total PD-L1 payload of platelets (pPD-L1 ${ }^{\text {Adj.) }}$ without the need of artifact prone in vitro stimulation procedures. Strikingly, in our study $\mathrm{pPD}-\mathrm{L} 1^{\mathrm{Adj}}$. is shown to be superior in predicting response to ICI when compared to immunohistochemistry-based quantification of PD-L1 on tumor biopsies.

\section{Results}

Tumor cells transfer PD-L1 to platelets. To address whether the immune regulatory protein PD-L1 can be transferred from tumor cells to platelets, we co-incubated platelets obtained from healthy donors with four different NSCLC tumor cell lines harboring varying expression levels of PD-L1 (NCI-H23, A549: PD-L1 low/ negative, NCI-H226, NCI-H460: PD-L1 positive) (Fig. 1a, b). PDL1 positivity was determined by flow cytometry and defined as PD-L1 expression in $\geq 5 \%$ of all tumor cells. PD-L1 expression on platelets (pPD-L1) was observed after co-incubation with the PDL1 expressing NCI-H226 and NCI-H460 cells but not after coincubation with the PD-L1 low/negative cell lines NCI-H23 and A549 (Fig. 1c-e). Results were validated using a flow cytometrybased approach (Fig. 1f). Co-incubation of platelets with all tumor cell lines resulted in platelet activation, as indicated by P-selectin (CD62P) induction (Fig. 1g), however only coincubation with PD-L1 positive NCI-H226 and NCI-H460 cells resulted in an increased PD-L1 expression on the platelet surface (Fig. 1h). To ensure that platelets from healthy donors, used in this assay, do not harbor relevant amounts of endogenous PD-L1 we conducted western blot analyses on platelet whole-cell lysates. Indeed, Western Blot data confirmed that platelets from healthy donors do not express relevant PD-L1 levels (Supplementary Fig. 5b).

Of note, conditioned medium from tumor cells induced platelet activation but did not result in increased levels of PDL1 protein on the platelet surface (Supplementary Fig. 3c, d), suggesting that PD-L1 transfer from tumor cells to platelets is dependent on a direct cell-cell contact between both cell types. Of note, frequent interaction with platelets was not restricted to adherent tumor cells but could for example also be observed for non-adherent A549 lung cancer cells (Supplementary Fig. 3e, f).

To gain deeper insights into the interaction of platelets and lung cancer cells, we took advantage of a live-cell imaging platform, where platelets are added to the medium and circulate through an imaging chamber that contains human NSCLC cells. Real time video microscopy revealed distinct interactions of tumor cells and platelets (Fig. 1i, j and Supplementary Movie 1). Strikingly, platelets remained fully agile and re-entered the circulation after contacting the tumor cell membrane (Fig. $1 \mathrm{k}-\mathrm{j}$ and Supplementary Movies 1,2). These data suggest that platelets can re-circulate after tumor cell attachment and activation and are in line with studies by Cloutier and Michaelson et al. ${ }^{16,17}$.

While platelets are anuclear, protein translation from RNA can nevertheless occur within platelets ${ }^{16-18}$. We therefore set out to investigate whether PD-L1 expression in platelets depends on a transfer of PD-L1 protein from tumor cells to platelets or whether a transfer of PD-L1 mRNA with subsequent protein synthesis within the platelet is involved. Transfection of vectors encoding for PD-L1-GFP and FLAG-GFP fusion proteins into PD-L1 negative A549 cells (Fig. 1m-o) resulted in high numbers of GFP positive platelets upon co-incubation (Fig. 1p-s). As inhibition of protein translation in platelets by cycloheximide did not result in a reduction of PD-L1-GFP expression in platelets (Fig. 1t), our data suggest that PD-L1 protein transfer and not mRNA transfer is underlying the observed $\mathrm{pPD}-\mathrm{L} 1$ expression after interaction of platelets and tumor cells 19,20 .

While the transfer of PD-L1-GFP or FLAG-GFP was robustly observed across various NSCLC cell lines, we nevertheless noted differences in protein transfer efficacies. For example, platelets showed low levels of FLAG-GFP and PD-L1-GFP after coincubation with NCI-H322, NCI-H522 and NCI-H23 cells, while HOP-62 and HOP-92 cells displayed significantly higher protein transfer rates (Fig. 2a-d). Given that our data indicated that a platelet-tumor-cell contact is necessary for a sufficient transfer of PD-L1 from tumor cells to platelets, we hypothesized that expression levels of adhesion molecules might determine the efficacy of protein transfer from tumor cells to platelets. Along these lines we found that PD-L1 transfer rates positively correlated with fibronectin (FN1) mRNA expression levels, while no significant correlation was found for fibrinogen alpha chain (FGA) or tissue factor (F3) mRNA expression (Fig. 2e-g). Of note, fibronectin expression also correlated with platelet-tumor cell interaction in vitro (Fig. $2 \mathrm{~h}-\mathrm{j}$ ). Immunofluorescence staining as well as analysis of protein-protein interaction via proximity ligation assay (PLA) revealed close proximity of fibronectin and PD-L1 (Fig. 2k-n) at the cell surface. In line with these observations, we found that siRNA mediated knockdown of fibronectin resulted in a significant reduction of PD-L1 transfer, thus functionally validating fibronectin as a key regulator of protein transfer from tumor cells to platelets (Fig. 2o-q). 


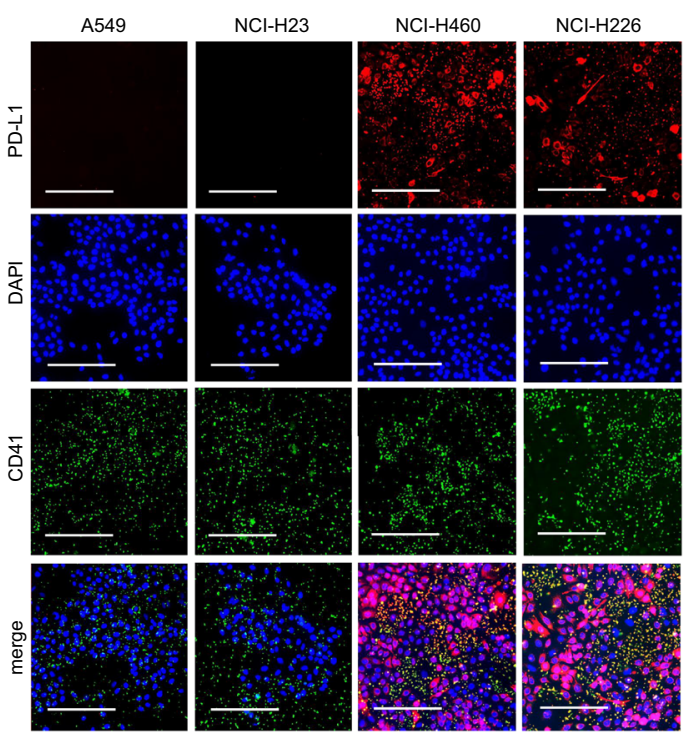

b
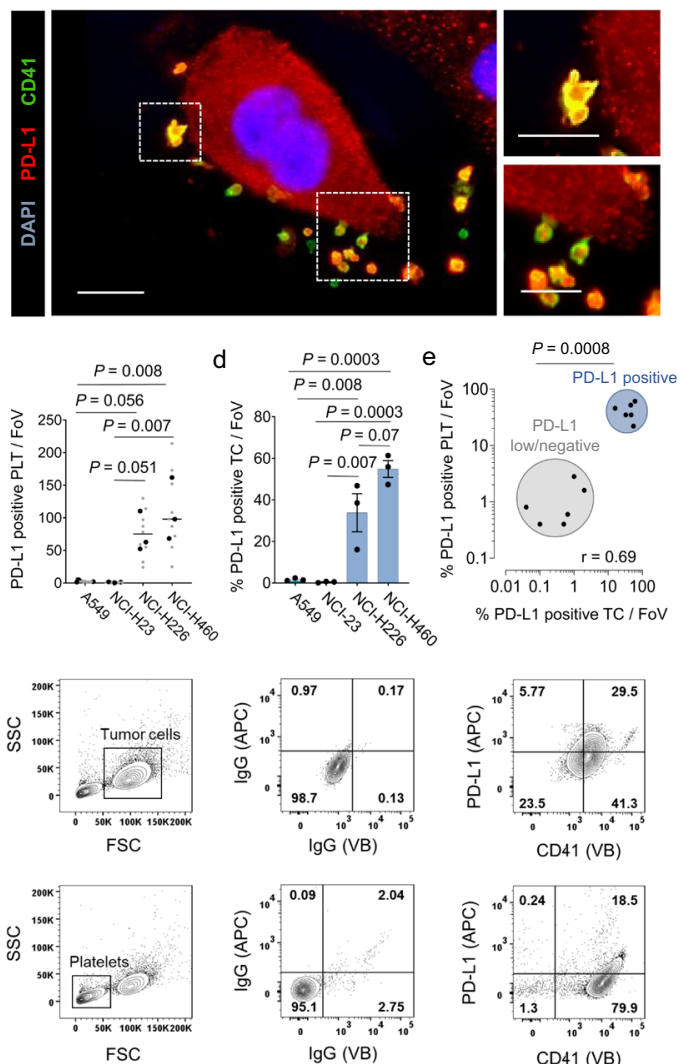

g

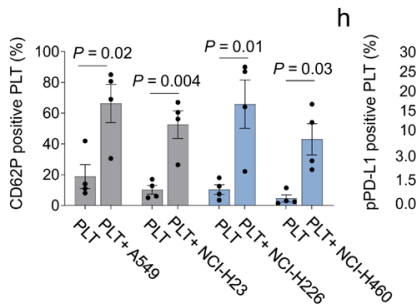

30

30
$25-$
$20-$

$15-10$.

${ }_{10} P=0.1 P=0.09$

1.5.

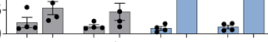

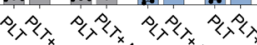

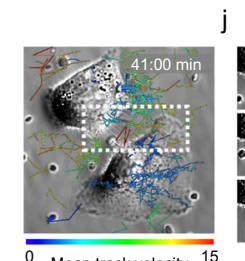

0 Mean track velocity 15

$\mathrm{m}$
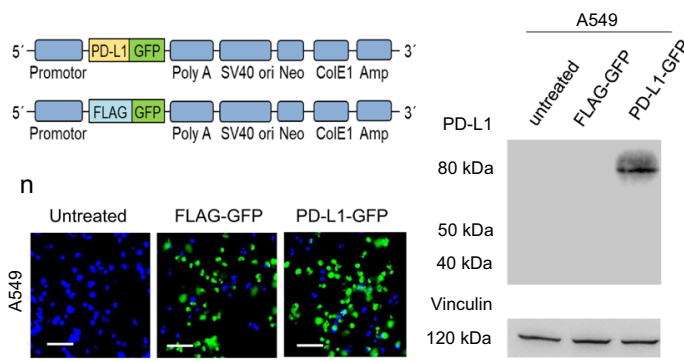

$\mathrm{p}$
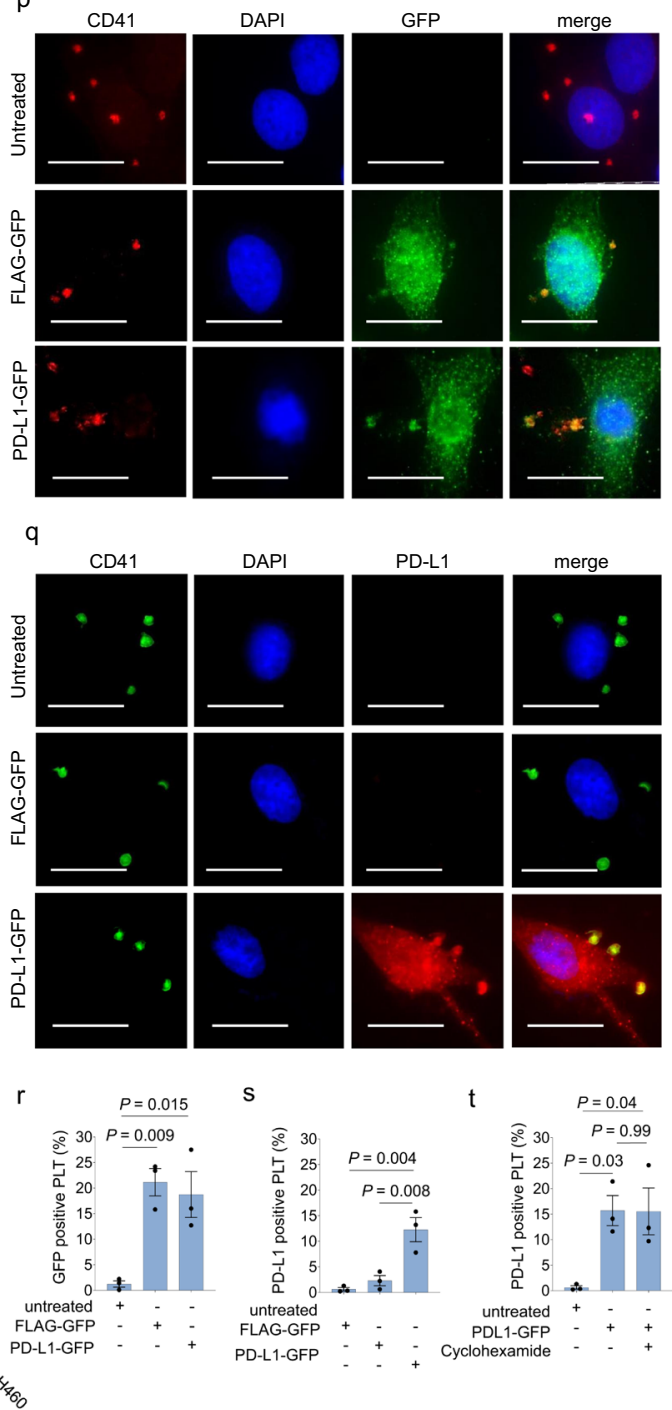

Platelet adhesion to fibronectin is known to be mediated via several molecules including GPIba, integrin $\alpha_{5} \beta_{1}$ or GPIIbIIIa $^{21,22}$. We therefore set out to address whether inhibition of these adhesion molecules on platelets reduces adhesion to fibronectin and PD-L1 uptake from tumor cells.
Strikingly, while monoclonal antibodies against GPIba and integrin $\alpha_{5} \beta_{1}$ prevented platelet adhesion to fibronectin (Fig. 2r-t) and PD-L1 protein transfer (Fig. 2u, v), inhibition of GPIIbIIIa by Tirofiban only marginally reduced platelet adhesion (Fig. 2r-t). 
Fig. 1 Direct platelet-tumor cell interactions result in PD-L1 expression on the platelet surface. a Representative immunofluorescence staining of PD-L1 (red)and the platelet marker CD41 (green) in four different NSCLC tumor cell lines (A549, NCl-H23, NCl-H460, NCl-H226) co-incubated with human platelets ( $n=3$ biological replicates). Scale bars $200 \mu \mathrm{m}$. b Immunofluorescence microscopy of $\mathrm{NCl}-\mathrm{H} 460$ cells interacting with human platelets derived from a healthy donor (PD-L1: red, CD41: green) $(n=3)$. Scale bar left $20 \mu \mathrm{m}$, right $10 \mu \mathrm{m}$. c Quantitative analysis of the PD-L1+ platelets per field of view (FoV), analyzed by immunofluorescence microscopy ( $n=9$ FoV (small symbols) were analyzed out of a total of $n=3$ independent experiments (large symbols)). Horizontal lines represent means. d Percentage of PD-L1+ tumor cells per FoV $(n=3)$. Data are mean \pm SEM. e Correlation of \% PD-L1+ platelets/FoV vs. \% PD-L1 tumor cells/FoV $(n=3)$. Correlation was determined by simple linear regression analysis. f Flow cytometry gating strategy for the quantification of PD-L1+ tumor cells (upper) and platelets (lower) after co-incubation. $\mathbf{g}$, $\mathbf{h}$ Surface expression of PD-L1 and CD62P on control platelets (PLT) and platelets after co-incubation with $\mathrm{A} 549, \mathrm{NCl}-\mathrm{H} 23, \mathrm{NCl}-\mathrm{H} 226, \mathrm{NCl}-\mathrm{H} 460$ cells $(n=4)$. Data are mean $\pm \mathrm{SEM}$. Statistical significance was calculated by two-tailed Student's $t$ test. i Phase-contrast image of A549 tumor cells after 41 min coculture with platelets (ratio 1:1000). Overlaid migration tracks were color-coded based on their mean velocity. $\mathbf{j}$ Image sequence depicting tumor cell interaction and protrusion of a single platelet followed by detachment derived from zoom-in area indicated in (i). $\mathbf{k}$ Percentage of stable platelet-tumor cell contacts lasting from contact initiation until the end of the observation period (total observation time: $41 \mathrm{~min}$ ). I Contact duration of platelet-tumor cell interactions. Data derived from the analysis of $n=75$ platelets out of one independent experiment. Boxes represent median and 25th to 75th percentiles, whiskers are minimum to maximum. $\mathbf{m}$ Scheme of vectors expressing PD-L1-GFP and FLAG-GFP used for transfection. $\mathbf{n}$ Immunofluorescence stainings of GFP of A549 cells transfected with FLAG-GFP or PD-L1GFP $(n=3)$. Scale bar $50 \mu \mathrm{m}$. o Western blot analysis for PD-L1 in untreated and transfected A549 cells $(n=2)$. Vinculin was used as loading control. Presentation of full scan blots are provided in the Source data file. p, q Representative immunofluorescence microscopy of untreated, FLAG-GFP and PD-L1GFP-transfected A549 cells interacting with platelets $(n=3$ ). Tumor cells and platelets were stained for GFP (upper) and PD-L1 (lower). Scale bar $50 \mu$ m. $\mathbf{r}$, s Flow-cytometry-based quantification of GFP $(\mathrm{I})$ and PD-L1 $(\mathbf{m})$ on platelet surfaces after co-incubation with untreated and transfected A549 cells $(n=3)$. $\mathbf{t}$ Expression of PD-L1 on platelets pre-treated with $100 \mu \mathrm{M}$ cycloheximide after co-incubation with PD-L1-GFP-transfected A549 cells ( $n=3$ ). $\mathbf{r}$-t Data are mean \pm SEM. c, d, $\mathbf{r}-\mathbf{t}$ Statistical significance was calculated by one-way ANOVA and Tukey's multiple comparisons test. Source data are provided as a Source Data file.

Detection of functional PD-L1 on platelets of NSCLC patients. To address the significance of our findings for human cancers, we next quantified PD-L1 expression on platelets in healthy lung tissue or NSCLC tumor tissue. While platelets were detected in high abundance in healthy lung tissue and PD-L1 negative NSCLC, we could not observe any relevant PD-L1 expression on these platelets (Fig. 3a, b, d, e). In contrast PD-L1 positive platelets were observed in high abundance in tissue sections from patients suffering from PD-L1 positive NSCLC (Fig. 3c-e). To quantify the number of PD-L1 positive platelets outside the tumor, we next isolated platelets from the peripheral blood of a cohort of 64 healthy donors and 128 NSCLC patients. Fluorescence-Activated Cell Sorting (FACS) revealed threefold higher numbers of PD-L1 positive platelets in NSCLC patients as compared to healthy donors (median pPD-L1 expression in healthy donors 0.29 (95\%CI: $0.21-0.44)$, median pPD-L1 expression in NSCLC patients 0.89 (95\%CI: 0.61-1.21) (Fig. $3 \mathrm{f}$ ). The detected differences were even higher, when total pPD-L1 levels were determined using a quantitative enzyme-linked immunosorbent assay (ELISA). While platelet rich plasma (PRP) from NSCLC patients in average contained $108.3 \mathrm{pg} / \mathrm{mL}$ PD-L1, PRP from healthy volunteers only contained $1.8 \mathrm{pg} / \mathrm{mL}$ (Fig. 3g, h). Differences in pPD-L1 expression in NSCLC patients versus healthy volunteers were also confirmed using western blot analysis (Supplementary Fig. 5b-d). Interestingly, PD-L1 expression was highest in patients with advanced (UICC stage IV) tumors (Supplementary Fig. 5e). Of note, immunofluorescence (Fig. 3i) and immunoelectron microscopy (Fig. 3j) revealed frequent $\mathrm{PD}-\mathrm{L} 1$ clusters in platelets obtained from peripheral blood of a PD-L1 positive NSCLC patient, further underlining functionality of $\mathrm{pPD}-\mathrm{L} 1$, as immune ligand clustering has been described to be a prerequisite for proper binding to its receptor $^{23}$.

Prompted by these results, we next explored whether pPD-L1 exerts immune-inhibitory functions. We stimulated human $\mathrm{T}$ cells from healthy donors with EBV/CMV-derived peptides in the presence or absence of PD-L1 positive platelets obtained from NSCLC patients. T cell activation was evaluated using an enzyme-linked-immuno-Spot (ELISpot) assays determining the effector cytokines IFNy and TNFa. In line with published data we observed that platelets dampen $\mathrm{T}$ cell activity independent of their PD-L1 expression status (Fig. $4 \mathrm{a}-\mathrm{c}$ and Supplementary Fig. $6 a, b)^{24,25}$. However, when PD-L1 expressing platelets were pre-treated with the anti-PD-L1 mAb Atezolizumab their T cell inhibitory effect was abolished (Fig. 4a-c). Next, we expanded our work towards tumor-associated antigens. New York esophageal squamous cell carcinoma 1 antigen (NY-ESO-1) belongs to the family of cancer-testis antigens, but is also aberrantly expressed in many tumor entities including NSCLC ${ }^{26}$. Stimulation of T cells from healthy donors with NY-ESO-1 peptides predominantly resulted in a clonal expansion of NY-ESO-1 specific CD4 ${ }^{+} \mathrm{T}$ cells (CD62L-/CD45RO + and CD27-/CD28+) (Fig. 4d), which were further specified as CD4+ effector memory $\mathrm{T}$ cells $\left(\mathrm{T}_{\mathrm{EM}}\right.$, CD62L-/CD45RO+ and CD27-/CD28+) (Fig. 4d, e). Remarkably, $\mathrm{T}_{\mathrm{EM}}$ activity, as determined by IFN $\gamma$ and $\mathrm{TNF} \alpha$ release, decreased significantly upon co-incubation with PD-L1 positive platelets. However, T cell activity could be restored when pPD-L1 positive platelets were pre-treated with anti-PD-L1 (Fig. 4f-k).

To investigate a potential impact of PD-L1 positive platelets on other immune cells, we also characterized changes in the overall immune cell composition (peripheral blood) in 10 NSCLC patients and five healthy controls (Supplementary Fig. 7a). In NSCLC patients pPD-L1 tended to be correlated negatively with the total number of NK $(p=0.1), \mathrm{CD} 4+\mathrm{T}$ cells $(p=0.09)$ and CD8+ T cells $(p=0.02)$ (Supplementary Fig. 7b). Moreover, in NSCLC patients more PD-1 and PD-L1 was expressed on dendritic cells (DCs), natural killer (NK) cells and CD4+ and CD8 $+\mathrm{T}$ cells (Supplementary Fig. 7c, d). In our analyses we did not observe a correlation of PD-1 or PD-L1 expression and pPDL1 in DCs, NK cells or CD4+ T cells (Supplementary Fig. 7e, f). However, we detected a positive correlation of $\mathrm{PPD}-\mathrm{L} 1$ and PD-1 on CD8+ T cells $(p=0.02)$. We also quantified T cells and T cell infiltration in the TME in eleven NSCLC patients with different levels of pPD-L1 using the MACSima ultradeep tissue profiling platform. Noteworthy, in patients with high PPD-L1 we observed lower numbers of $\mathrm{T}$ cells in the TME and less infiltrating T cells (Fig. 5a-d). In contrast to our findings in the peripheral blood, we observed an inverse correlation of PD-1 on T cells and pPD-L1 (Fig. 5e, f).

Regulation of pPD-L1 during platelet activation. As it is well established that expression levels of platelet surface proteins 

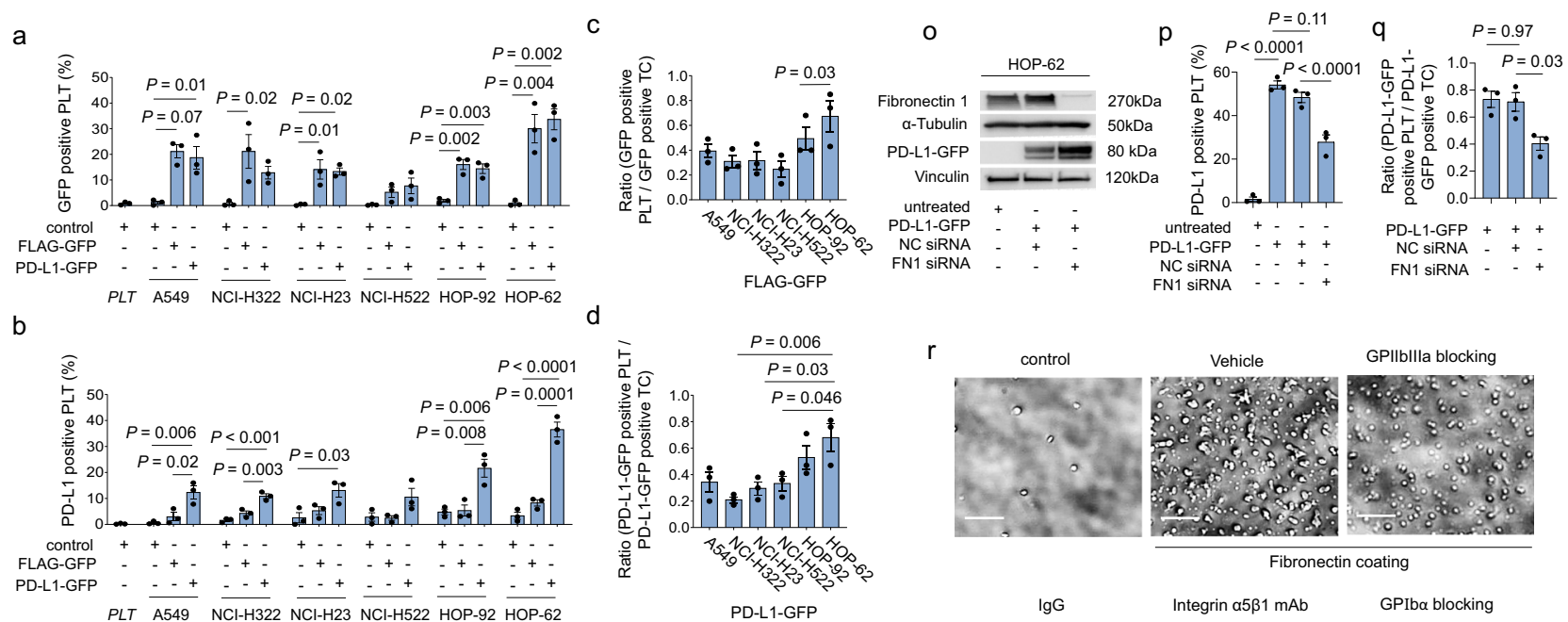
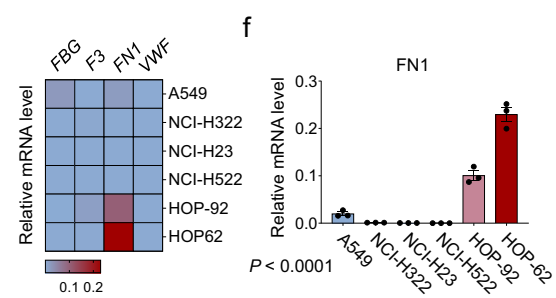

h

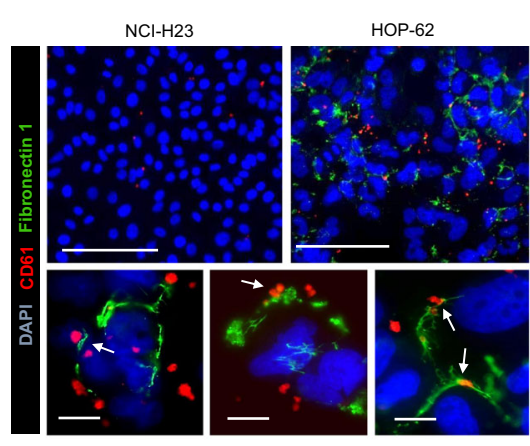

$\mathrm{k}$

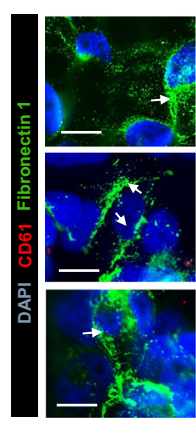

HOP-62
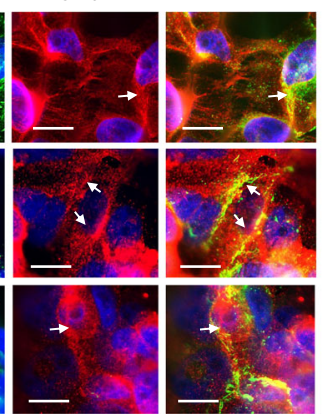

g

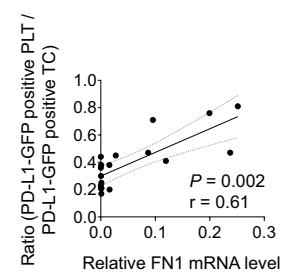

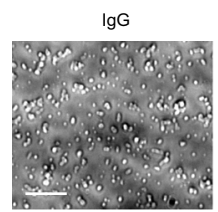

Integrin $\alpha 5 \beta 1 \mathrm{mAb}$

GPIb $\alpha$ blocking

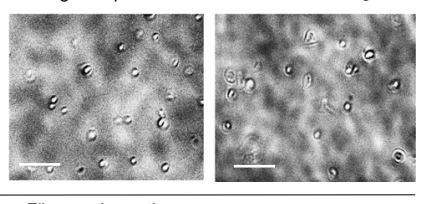

Fibronectin coating
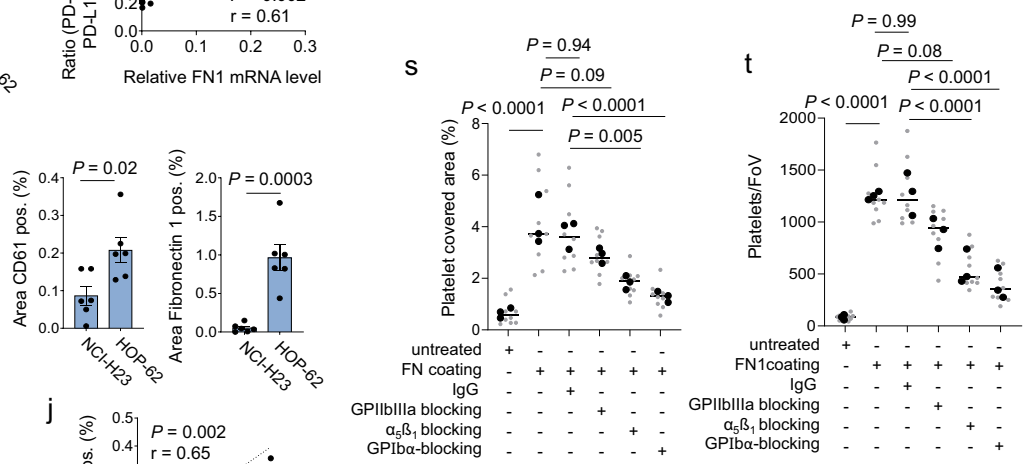

$\mathrm{u}$
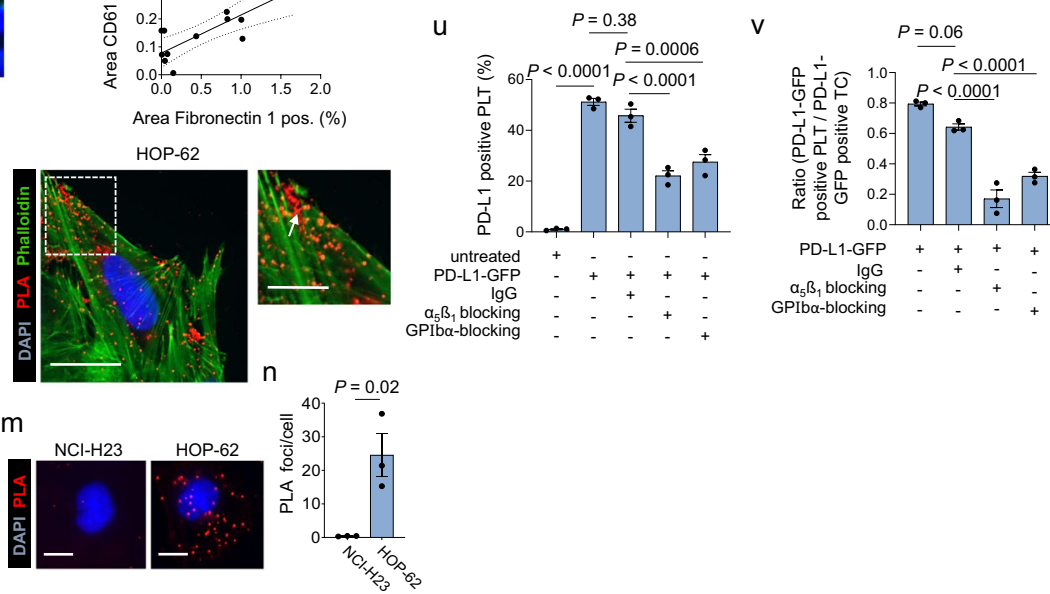

correlate with the platelet activation status, we reasoned that different degrees of platelet activation might underlie varying levels of pPD-L1 expression on the platelet surface. Indeed, when we analyzed the platelet activation marker CD62P, we observed varying CD62P expression levels which showed a strong positive correlation with pPD-L1 expression (Fig. 6a). Of note, while PD-L1 expression in general was lower in unstimulated platelets, we were able to robustly detect pPD-L1 on the platelet surface of resting (CD62P negative) platelets (Supplementary Fig. 5a). In line with this, we also detected pPD-L1 in $\alpha$-granules (Fig. 6b).
As even highly standardized blood collection procedures can result in varying levels of shear-stress mediated platelet activation and therefore complicates standardization ${ }^{27,28}$, we hypothesized that different levels of platelet pre-activation might complicate the interpretability and comparability of pPD-L1 levels on freshly collected platelets from different patients. We therefore reasoned that a controlled in vitro activation of platelets with subsequent maximization of pPD-L1 expression might most adequately uncover the total payload of platelet PD-L1 and best possibly allow a comparison between different patients. Indeed, we found that PPD-L1 expression was maximized upon controlled platelet 
Fig. 2 Fibronectin mediates platelet adhesion to tumor cells and facilitates PD-L1 protein transfer. $\mathbf{a}$, b Expression of GFP (a) and PD-L1 (b) on platelet surfaces after co-incubation with six FLAG-GFP- and PD-L1-GFP--transfected NSCLC cell lines (A549, NCl-H322, NCl-H522, NCl-H23, HOP-62, HOP-92) $(n=3)$. c, d Ratio of GFP+ platelets/GFP+ tumor cells (c) and PD-L1-GFP+ platelets/PD-L1-GFP+ tumor cells (d) after co-incubation of platelets with transfected NSCLC cell lines $(n=3)$. e Heat map of relative fibrinogen (FBG), tissue factor (F3), fibronectin 1 (FN1) and von Willebrand factor (VWF) mRNA levels in all tested NSCLC cell lines $(n=3)$. $\mathbf{f}$ Relative mRNA level of $F N 1$ in all tested NSCLC cell lines $(n=3)$. $\mathbf{g}$ Correlation ratio of PD-L1-GFP+ platelets/ PD-L1-GFP+ tumor cells and relative FN1 mRNA level $(n=3) . \mathbf{h}$ Immunofluorescence images of platelet adhesion to NCI-H23 (upper left) and HOP-62 cells (upper right and lower) (fibronectin: green, platelets: red) $(n=3)$. Upper scale bar $100 \mu \mathrm{m}$, lower $20 \mu \mathrm{m}$. $\mathbf{i}$ Left, Quantification of adhesive platelets after co-incubation with $\mathrm{NCl}-\mathrm{H} 23$ and HOP-62. Quantified as CD61+ area in \%/FoV ( $n=6$ out of 3 independent experiments). Right, quantification of fibronectin covered area in \%/FoV in NCl-H23 and HOP-62 cells ( $n=6$ out of 3 independent experiments). $\mathbf{j}$ Correlation of platelet and fibronectin covered area in \%/FoV. $\mathbf{k}$ Immunofluorescence images of PD-L1 and fibronectin expression in HOP-62 cells $(n=2)$. Scale bar $20 \mu \mathrm{m}$. I Representative PLA with PD-L1 and fibronectin in HOP-62 cells. Scale bar left $20 \mu \mathrm{m}$, right $10 \mu \mathrm{m}(n=1)$. $\mathbf{m}$ Representative PLA with PD-L1 and fibronectin in NCI-H23 and HOP-62 cell $(n=3)$. Scale bar $10 \mu \mathrm{m}$. $\mathbf{n}$ PLA quantification of foci/cell in $119 \mathrm{NCl}-\mathrm{H} 23$ and $126 \mathrm{HOP}-62$ cells out of three biological replicates. $\mathbf{o}$ Western blot for PD-L1 and fibronectin in PD-L1-GFP-transfected HOP-62 cells after siRNA knockdown for fibronectin. Vinculin and $\alpha$-Tubulin were used as loading controls $(n=2)$. Presentation of full scan blots are provided in the Source data file. $\mathbf{p}$ Expression of PD-L1 on platelets after co-incubation with PD-L1-GFP, PD-L1-GFP/siFN1, and PD-L1-GFP/siNC-transfected HOP-62 cells $(n=3)$. q Ratio PD-L1-GFP+ platelets/PD-L1-GFP+ tumor cells after co-incubation of platelets with PD-L1-GFP, PD-L1-GFP/siFN1, and PD-L1-GFP/siNC-transfected HOP-62 cells $(n=3)$. $\mathbf{r}$ Representative images of platelet adhesion to fibronectin-coated surface in the presence or absence of different platelet-blocking agents $(n=3)$. Scale bar $20 \mu \mathrm{m}$. $\mathbf{s}$, $\mathbf{t}$ Quantitative analysis of the platelet adhesion assay as platelet covered area/FoV in \% ( $)$ and platelets/FoV ( $t$ ) ( $n=9$ (small symbols) were analyzed out of three independent experiments (large symbols)). Horizontal lines represent mean. Statistical significance was calculated by one-way ANOVA and Tukey's multiple comparisons test. u Quantification of PD-L1 on platelets after co-incubation with PD-L1-GFP-transfected HOP-62 cells with or without pre-treatment with platelet-blocking agents $(n=3)$. v Ratio PD-L1-GFP+ platelets/PD-L1-GFP+ tumor cells after co-incubation of platelets with PD-L1-GFP-transfected HOP-62 cells with or without pre-treatment with platelet-blocking agents $(n=3)$. $\mathbf{a}-\mathbf{d}, \mathbf{f}, \mathbf{p}, \mathbf{q}, \mathbf{u}, \mathbf{v}$ Data are mean \pm SEM. Statistical significance was calculated by one-way ANOVA and Tukey's multiple comparisons test. $\mathbf{g}$, j Correlation was determined by simple linear regression analysis. I, $\mathbf{n}$ Data are mean \pm SEM. Statistical significance was calculated by two-tailed Student's $t$ test. Source data are provided as a Source Data file.

stimulation with the PAR1 agonist TRAP-6 (Fig. 6c-f and Supplementary Fig. 8 a-d) or other platelet activation agents such as ADP or collagen (Fig. 6g, h) and thus might allow for a better comparability of pPD-L1 levels between different patients. However, controlled platelet activation and subsequent measurement of CD62P and pPD-L1 is technically demanding and might prevent the use of $\mathrm{pPD}-\mathrm{L} 1$ as a biomarker in clinical routine. We therefore set out to explore whether a normalized PD-L1 level on the platelet surface could be calculated without in vitro manipulation of platelets. To do so we developed an adjustment model based on the calculation of $\triangle \mathrm{PD}$-L1 (ratio of PD-L1 before and after stimulation) as a function of PPD-L1 expression in unstimulated platelets and the degree of pre-activation (CD62P expression) (Fig. 6i, j). Specifically, we devised a matrix which allows us to calculate PD-L1 Adj. for subgroups of patients harboring different levels of platelet pre-activation (CD62P expression) and pPD-L1 expression (Fig. 6j and Supplementary Fig. 9). Taking advantage of our matrix, corrected PD-L1 levels, designated PPD-L1 ${ }^{\text {Adj. }}$, were determined for all patients (Fig. 6k).

Adjusted platelet-derived PD-L1 serves as a prognostic and predictive marker in NSCLC. We first used the calculated PPD$\mathrm{L} 1{ }^{\text {Adj. }}$ levels and performed a receiver-operating characteristic (ROC) analysis for overall survival (OS). We found that pPD$\mathrm{L}^{\text {Adj. }}$ levels in the subgroup of maximal platelet activation (CD62P 80-100\%) showed highest accuracy in predicting OS and were superior in predicting OS compared to pPD-L1 (Fig. 7a). Since no cut-off value for $\mathrm{pPD}-\mathrm{L} 1{ }^{\text {Adj }}$ had been established so far, we analyzed OS in pPD-L1 Adj. quartile groups (Q1-4) using the Kaplan-Meier method (Fig. 7b, c). Details regarding characteristics of our patient population are provided in Supplementary Table 1. The median observation time for monitoring OS in our study was 23.5 months (95\%CI: 3.4-67.55 months). At data cutoff for overall survival, 42 of 128 patients $(32.8 \%)$ were still alive. Strikingly, patients with high pPD-L1 Adj. levels showed a significantly shortened OS (Fig. 7b). The median survival in Q1 (low pPD-L1 Adj. levels) was 43 months compared to only 24 months in

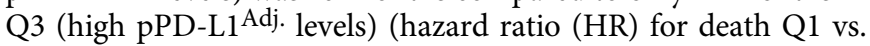
Q3: 2 (95\%CI: 1-3.9)) and 14 months in Q4 (very high pPD-

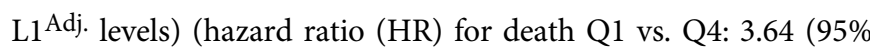
CI: 1.97-6.72)). Importantly, the observed differences in OS were not restricted to the time since initial diagnosis but were still significant when analyzing the time period since platelet analysis (Fig. 7c)

It has been reported that mutations in key oncogenic drivers do not only fuel proliferation via cell intrinsic cues but also impact tumor biology via modulation of the tumor microenvironment ${ }^{29-31}$. Along these lines, we found increased PPD-L1 Adj. levels in patients suffering from KRAS mutated NSCLC as compared to those with KRAS wildtype status (Fig. 7d). In contrast, mutations in EGFR, ALK fusions and ROS1 fusions or mutations showed no association with

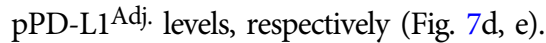

We also explored a potential correlation of $\mathrm{pPD}$-L1 ${ }^{\text {Adj. }}$ with other clinical parameters. For example, we found that patients with higher tumor stages ( $\mathrm{T}, p=0.03$ ), higher degrees of lymph node invasion $(\mathrm{N}, p=0.04)$ and a higher tumor grading $(\mathrm{G}$, $p=0.002)$ expressed more PD-L1 on the platelet surface (Fig. $7 \mathrm{f}-\mathrm{h}$ ). No association was found between pPD-L1 Adj. and the region of tumor origin (central vs. peripheral, Fig. 7i). However, pPD-L1 ${ }^{\text {Adj. }}$ strongly correlated with the occurrence of metastases $(p<0.001)$, especially liver $(p=0.005)$ and bone metastasis $(p=0.001)$ (Fig. $7 \mathrm{j}-1)$. In line with previous studies ${ }^{32}$, we also found $\mathrm{pPD}-\mathrm{L1} 1^{\text {Adj. }}$ to be positively correlated with smoking history and the amount of pack years (Supplementary Fig. 10c, d). Moreover, pPD-L1 ${ }^{\text {Adj. }}$.was positively correlated with platelet count, LDH and CRP (Supplementary Fig. 10j-1).

To further elaborate on the potential of $\mathrm{pPD}-\mathrm{L} 1^{\mathrm{Adj}}$ as a predictive biomarker in NSCLC, we conducted sequential measurements of $\mathrm{PPD}-\mathrm{L1}^{\mathrm{Adj}}{ }^{\mathrm{A}}$ in 12 patients undergoing conventional chemotherapy or ICI (Supplementary Fig. 11a, b). Details on therapeutic regimens are provided in Supplementary Fig. 4. In these patients baseline $\mathrm{PPD}-\mathrm{L} 1^{\mathrm{Adj}}$. levels were determined prior to the first cycle of the respective 1st line treatment. The second measurement was conducted in parallel to the first CT scan. In patients treated with a platinum-based chemotherapy the tumor responses were quantified according to the Response Evaluation Criteria in Solid Tumors (RECIST) guidelines ${ }^{33}$. For patients receiving ICI iRECIST guidelines ${ }^{34}$ were used. In both groups 
a

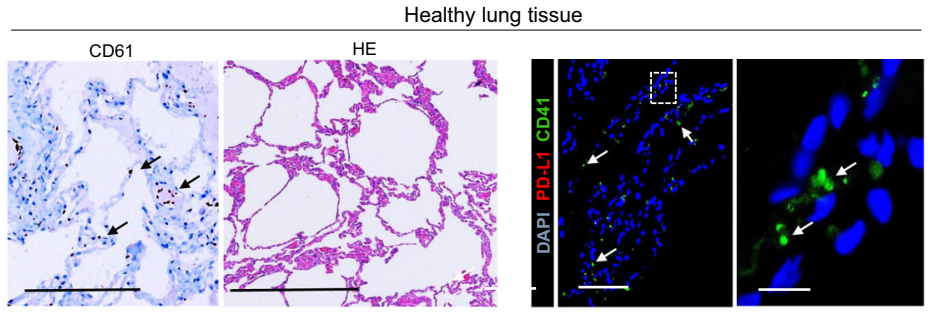

b

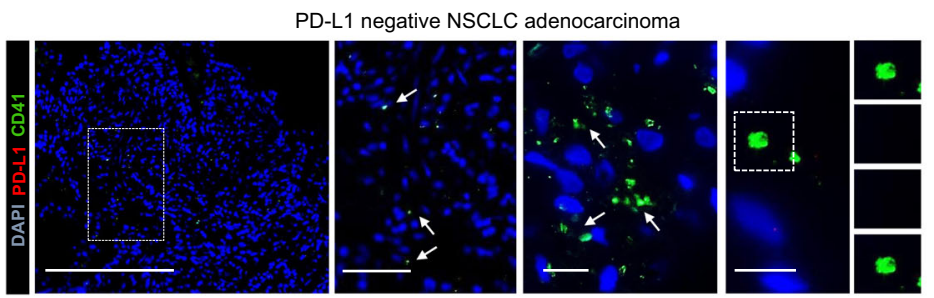

C

PD-L1 positive NSCLC adenocarcinoma

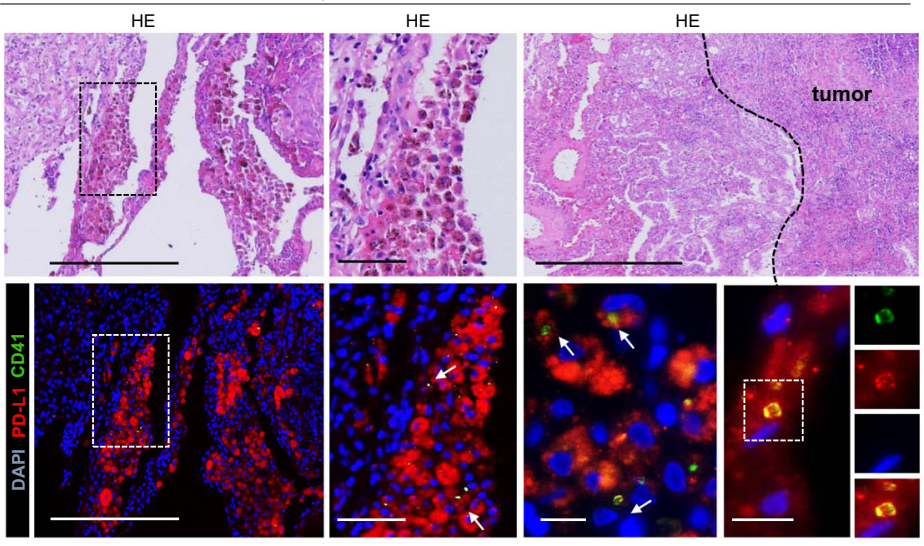

d

e
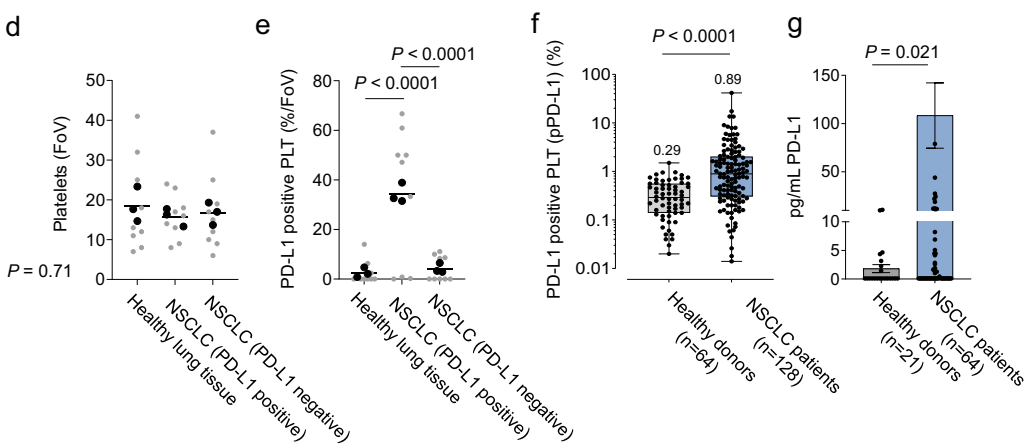

$\mathrm{h}$

i Platelets derived from peripheral blood

j Platelets derived
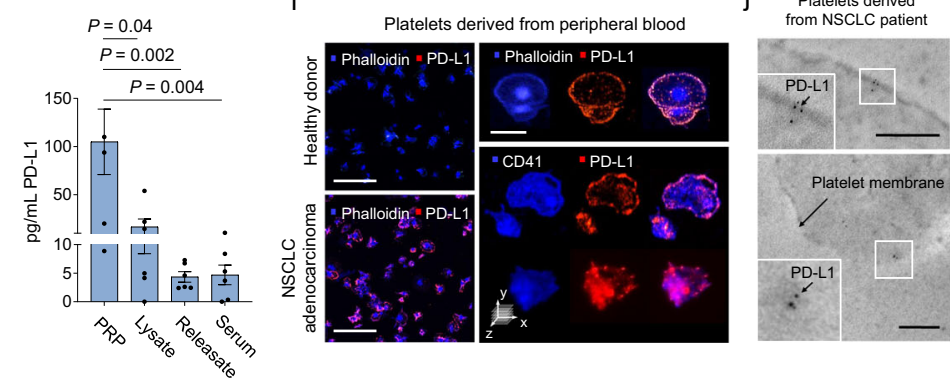

response evaluation was performed 6-8 weeks after initial treatment. Remarkably, a significant drop in pPD-L1 ${ }^{\text {Adj. }}$ levels was detected upon initiation of therapy in those patients whose tumors were later identified to have undergone at least partial remission (PR) ( $p=0.02$, Supplementary Fig. 11a). In contrast, patients who were later identified to have progressed despite therapy displayed a significant increase of PPD-L1 ${ }^{\text {Adj. }}$ already in early measurements after therapy initiation $(p=0.04$, Supplementary Fig. 11b). Of note, the predictive value of $\mathrm{pPD}-\mathrm{L1}^{\mathrm{Adj}}$. was robust regardless of the used therapeutic regime. In two patients

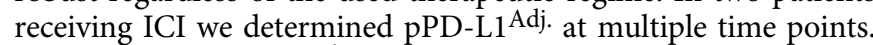
Remarkably, pPD-L1 ${ }^{\text {Adj. }}$ expression changes correlated with 
Fig. 3 Platelets from NSCLC patients show increased PD-L1 levels. a Left panel, left, Immunohistochemistry for CD61 in healthy human lung tissue (black arrow highlights CD61+ platelets). Scale bar $200 \mu \mathrm{m}$. Left panel, right, Representative micrograph of healthy lung tissue (H\&E). Scale bar $500 \mu \mathrm{m}$. Right panel, Immunofluorescence microscopy for CD41+ (green), PD-L1- (red) platelets in healthy lung tissue $(n=3)$. Scale bar left $100 \mu \mathrm{m}$, right $10 \mu \mathrm{m}$. b Immunofluorescence staining for CD41 (green) and PD-L1 (red) on platelets in a PD-L1- NSCLC patient tumor sample $(n=3)$. Scale bar left $500 \mu m$, center left $50 \mu \mathrm{m}$, center right and right $10 \mu \mathrm{m}$. c Upper, Representative micrographs of NSCLC adenocarcinoma (H\&E) ( $n=3)$. Scale bar left $250 \mu \mathrm{m}$, center $50 \mu \mathrm{m}$, right $500 \mu \mathrm{m}$. Lower, Immunofluorescence staining for CD41 (green) and PD-L1 (red) ( $n=3$ ). Scale bar left $500 \mu \mathrm{m}$, center left $50 \mu \mathrm{m}$, center right and right $10 \mu \mathrm{m}$. d, e Quantitative analysis of the platelets/FoV (d) and PD-L1+ platelets (\%/FoV) (e) in healthy lung tissue, PD-L1+ and PDL1- NSCLC patients $(n=9)$ (small symbols) were analyzed out of three independent experiments in three healthy donors and six NSCLC patients (large symbols). Horizontal line represent mean. f Percentage of PD-L1+ platelets in healthy donors $(n=64)$ and NSCLC patients $(n=128)$. Each dot represents a single donor. Boxes represent median and 25th to 75th percentiles, whiskers are minimum to maximum. $\mathbf{g}$ Total amount of PD-L1 (pg/mL) in healthy donors $(n=21)$ and NSCLC patients $(n=64)$ analyzed by ELISA. Protein level were analyzed in 64 out of 128 , randomly assigned patients of the NSCLC cohort. Data are mean \pm SEM. h Total amount of PD-L1 $(\mathrm{pg} / \mathrm{mL})$ in PRP, platelet lysate, platelet releasate, and serum $(n=6)$. Data are mean \pm SEM $\mathbf{i}$ Left, Representative PD-L1 immunofluorescence staining of platelets from a healthy donor (upper) and a NSCLC patient (lower). Platelets were counter stained with phalloidin. Right, Expression pattern of PD-L1 on a platelet derived from a NSCLC patient counter stained with phalloidin (upper) or CD41 (lower). Scale bar left $10 \mu \mathrm{m}$, right $2 \mu \mathrm{m}(n=1)$. j Platelets of a NSCLC patient, assessed by transmission electron microscopy. PD-L1 stained with post-embedding immunogold-labeling. PD-L1 gold particles densely accumulating on the platelet membrane (black dots). Upper scale bar $2 \mu \mathrm{m}$, lower $100 \mathrm{~nm}(n=1)$. $\mathbf{d}, \mathbf{e}, \mathbf{h}$ Statistical significance was calculated by one-way ANOVA and Tukey's multiple comparisons test. $\mathbf{f}$, $\mathbf{g}$ Statistical significance was calculated by Mann-Whitney test. Source data are provided as a Source Data file.

disease activity routinely determined via CT-scan (Supplementary Fig. 11c-f). As genomic alterations in EGFR and ALK represent independent factors influencing OS and progression-free survival (PFS), especially in patients receiving ICI, we additionally analyzed the role of PPD-L1 ${ }^{\mathrm{Adj}}$. in the respective subgroups with or without such alterations. Whereas we were not able to detect a significant difference regarding OS (Supplementary Fig. 12a-c), in $\mathrm{PPD}-\mathrm{L1} 1^{\text {Adj. }}$ high patients harboring an EGFR or ALK alteration who received a platinum-based chemotherapy, PFS tended to be worse compared to patients without EGFR and ALK alteration (Supplementary Fig. 12d, e). This might be explained by the fact that these patients had already shown a tumor progression upon first-line treatment with a tyrosine kinase inhibitor (TKI). In patients with EGFR and ALK alteration who received TKI, pPD-L1 Adj. was not predictive for PFS (Supplementary Fig. 12f). Since in our cohort none of the patients receiving ICI showed EGFR or ALK aberrations, the role of pPDL1 ${ }^{\text {Adj. }}$ could not be investigated in this cohort.

Finally, we set out to probe whether the pre-therapeutically determined PPD-L1 ${ }^{\text {Adj. }}$ level can predict the therapy response of NSCLC patients to immune-checkpoint blocking antibodies. To do so we analyzed the PFS of patients either treated with only conventional chemotherapy or immunocheckpoint blockade. pPD-L1 positive and negative subgroups were defined according to the median $\mathrm{PPD}-\mathrm{L} 1^{\mathrm{Adj}}$. level. In patients receiving conventional chemotherapy we observed a significantly higher PFS when pPDL1 levels were low (Fig. $7 \mathrm{~m}$ ). Interestingly, in patients treated with ICI (Pembrolizumab or Nivolumab), high pPD-L1 ${ }^{\text {Adj. }}$ predicted a PFS benefit (HR 4.74, $p=0.003$ ) (Fig. $7 \mathrm{n}$ and Supplementary Fig. 12j). Strikingly, when the predictive power of pPD-L1 ${ }^{\text {Adj. }}$ was compared to conventional histological PD-L1 quantification (TPS $>50 \%$ and $\geq 1 \%$ in tumor biopsies), pPD-L1 Adj. was found to much better predict therapy response towards ICI (Fig. 7o, Supplementary Fig. 12k, l). In summary, our data suggest that pre-therapeutically measured PPD-L1 Adj. levels accurately predict the therapeutic response towards immune-checkpoint blocking antibodies. Prospective clinical trials are warranted to validate our findings and to justify the implementation of pPD-L1Adj as a biomarker in clinical routine.

\section{Discussion}

Human cancers are heterogenous and biomarkers based on histopathological analyses of single tumor biopsies are often lacking robustness. Histological quantification of intratumoral PD-L1 expression is routinely performed on NSCLC biopsy material as an attempt to predict responses towards immune-checkpoint inhibition, however, the correlation between expression levels and the overall response rate (ORR) is limited ${ }^{12}$. In our present study we show that blood platelets are in frequent contact with lung cancer cells in vitro and in vivo and take up PD-L1 from the cancer cells in a fibronectin, integrin a5 $\beta 1$ and GPIb $\alpha$ dependent manner. Our data provides mechanistic explanation for recent reports describing $\mathrm{PD}-\mathrm{L} 1$ on platelets from patients suffering from different types of cancers $32,35,36$. Interestingly, while there is comprehensive literature describing tumor cell induced platelet aggregation (TCIPA) and tumor cell-associated thrombus formation $^{37-41}$, our herein presented data suggest that platelettumor cell contact can occur without substantial platelet activation and degranulation. Since pPD-L1 has not only been detected on the surface of activated platelets but also in resting platelets, it is tempting to speculate on an equilibrium between intracellularly stored PPD-L1 in a-granules and cell surface pPD-L1. Indeed, a similar mechanism has been described for the uptake and redistribution of fibrinogen and immunoglobulins ${ }^{42}$.

Importantly, as pPD-L1 is found to inhibit $\mathrm{T}$ cell function, it is likely that PPD-L1 plays a distinct role in systemic immunomodulation. Of note, pPD-L1 has recently been described in patients suffering from tumors which were classified as PD-L1 negative in biopsies $^{35}$. Our herein presented data as well as other published studies on tumor heterogeneity suggest that immunohistochemistrybased quantification of protein expression on tissue sections from single biopsies should be interpreted with caution, as protein expression might differ spatially and temporally ${ }^{43}$. Obviously, while our herein presented data suggest a highly efficient uptake of PD-L1 from lung cancer cells into platelets, it does not exclude that some pPD-L1 might be derived from other sources such as endothelial or other non-malignant cell types.

As the total blood volume is circulated up to 1000 times through the body each day, we reasoned that platelets might mirror the collective PD-L1 payload of a tumor and thus might open up venues for novel biomarker strategies. In this regard it is striking that pPD-L1 not only correlates with tumor stage/grade and the occurrence of metastases but is found to be superior in predicting response towards immune-checkpoint inhibition when compared to standard histological PD-L1 quantification on tumor biopsies. Since in particular lung cancer represents one of the most frequent and lethal cancers worldwide ${ }^{10}$, further clinical investigation of pPD-L1 as a biomarker in NSCLC does not only hold the promise to unburden our health systems by avoiding costly and unnecessary therapies with ICI but, even more 
a

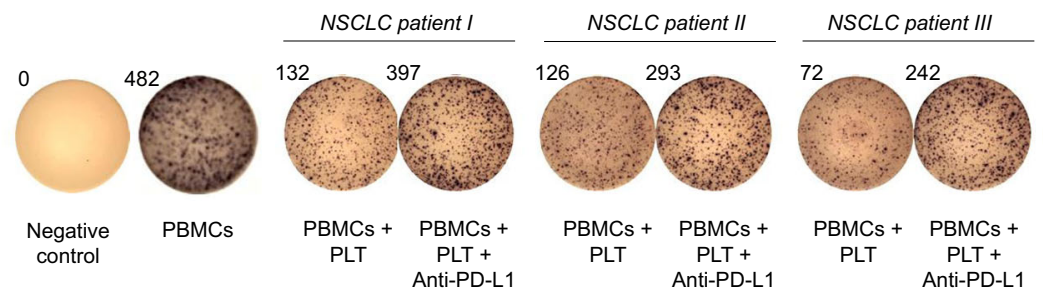

b

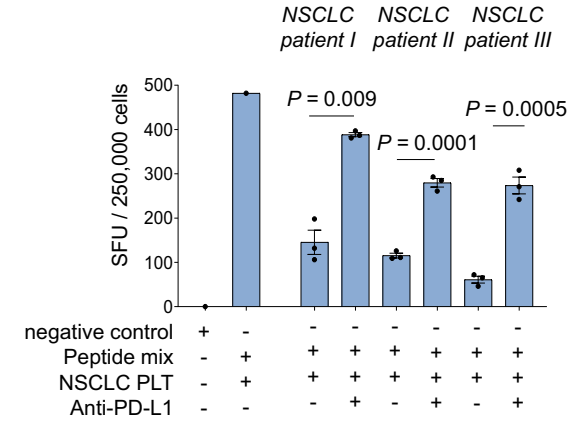

d

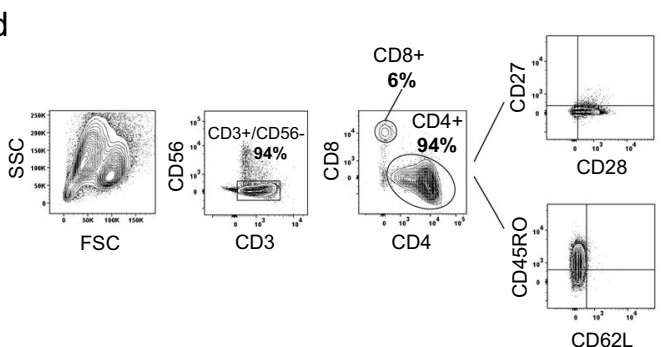

$f$

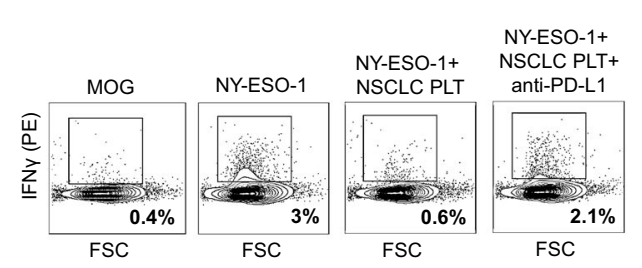

i

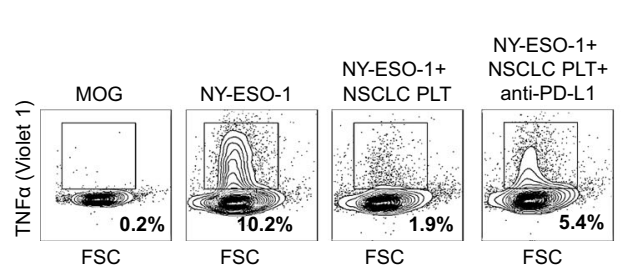

important, will avoid side effects of ICI in patients who would not benefit from this kind of therapy.

It should be mentioned that our exploratory study suffers from some limitations. Owing to the fact that we used an exploratory cohort of NSCLC patients with unequal representation of tumor stages and different cycles of various treatment regimens for the
C

NSCLC NSCLC NSCLC

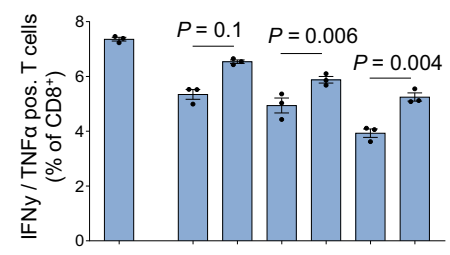

Peptide mix $+\quad++++++$ $\begin{array}{rlllllll}\text { NSCLC PLT } & - & + & + & + & + & + & + \\ \text { Anti-PD-L1 } & - & - & + & - & + & - & +\end{array}$

e

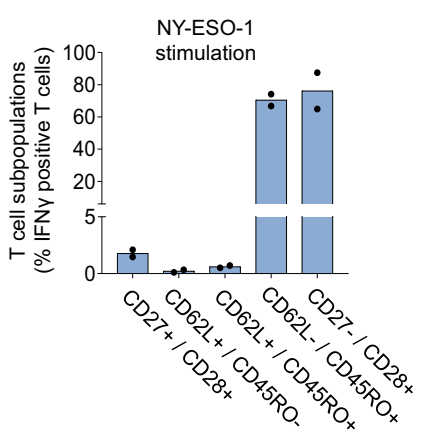

g
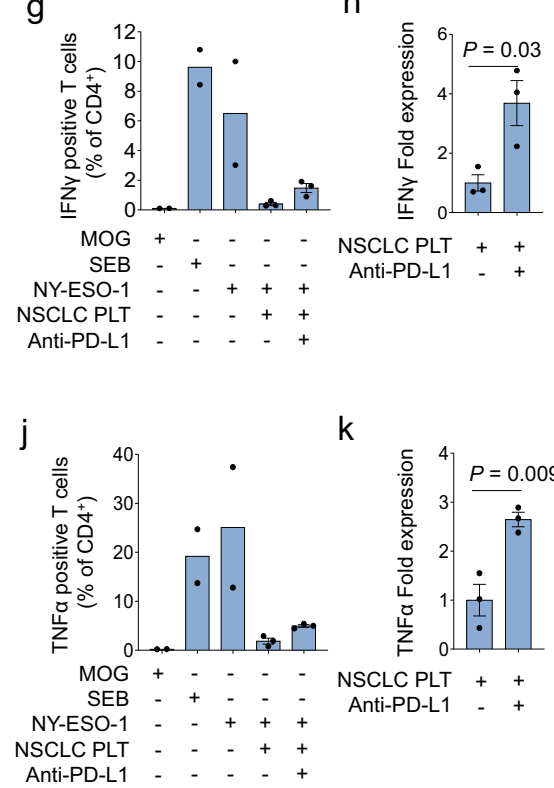
Fig. 4 PD-L1 on platelets shows functional relevance via decreasing T-cell activity. a IFN $\gamma$ ELISPOT assay of peptide-specific T-cells co-incubated with PDL1+ platelets with or without anti-PD-L1 mAb pre-treatment $(n=3)$. b Quantification of the IFN $\gamma$ ELISPOT assays $(n=3)$. c Flow cytometry-based quantification of indicated cytokines and surface markers for peptide stimulated CD8+ T-cells co-incubated with PD-L1+ platelets with or without anti-PD-L1 mAb pre-treatment $(n=3)$. d Representative FACS plots showing the gating strategy and the T-cell subpopulations after pre-sensitization, enrichment, and expansion. e Quantitative sub-phenotyping of NY-ESO-1 specific T cells using flow cytometry $(n=2)$. $\mathbf{f}$ Representative FACS plots displaying CD4+ TEM activity levels measured by INF $\gamma$ expression after co-incubation with PD-L1+ platelets with or without anti-PD-L1 mAb pre-treatment ( $n=3$ ). $\mathbf{g}$ Quantification of INF $\gamma+$ CD4 + TEM $(n=3)$. $\mathbf{h}$ IFNy fold change in CD4+ TEM $(n=3)$. i Representative FACS plots displaying CD4+ TEM activity levels measured by TNF $\alpha$ expression after co-incubation with PD-L1+ platelets with or without anti-PD-L1 mAb pre-treatment $(n=3)$. $\mathbf{j}$ Quantification of TNF $\alpha+C D 4+$ TEM $(n=3)$. $\mathbf{k}$, $\mathrm{TNF} \alpha$ fold change in CD4+ TEM $(n=3) . \mathbf{b}, \mathbf{c}, \mathbf{g}, \mathbf{h}, \mathbf{j}, \mathbf{k}$ Data are mean \pm SEM. Statistical significance was calculated by two-tailed Student's $t$ test. Source data are provided as a Source Data file.

a

$$
\begin{aligned}
& \text { NSCLC adenocarcinoma } \\
& \text { (pPD-L1 low) }
\end{aligned}
$$
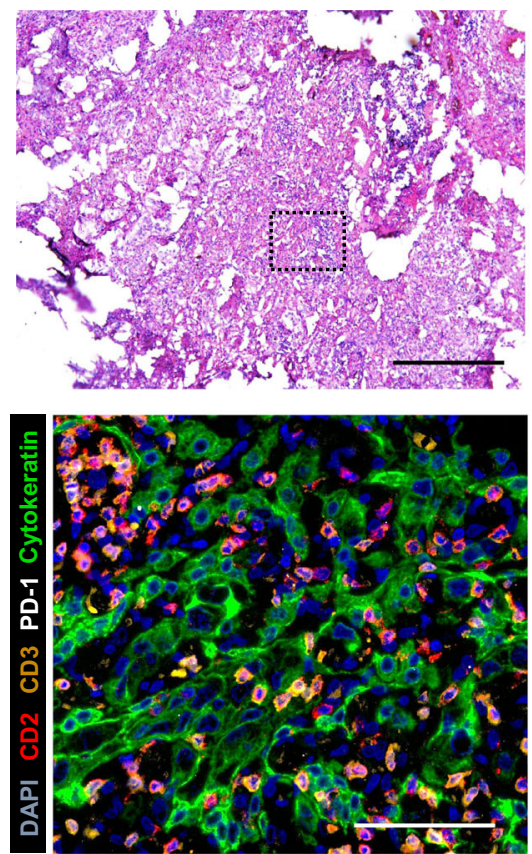

C

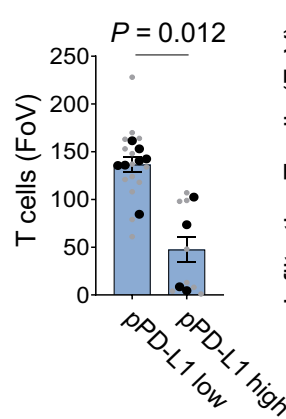

d

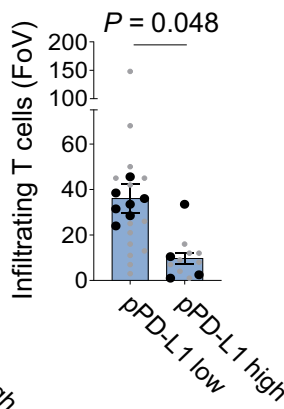

b

NSCLC adenocarcinoma (pPD-L1 high)
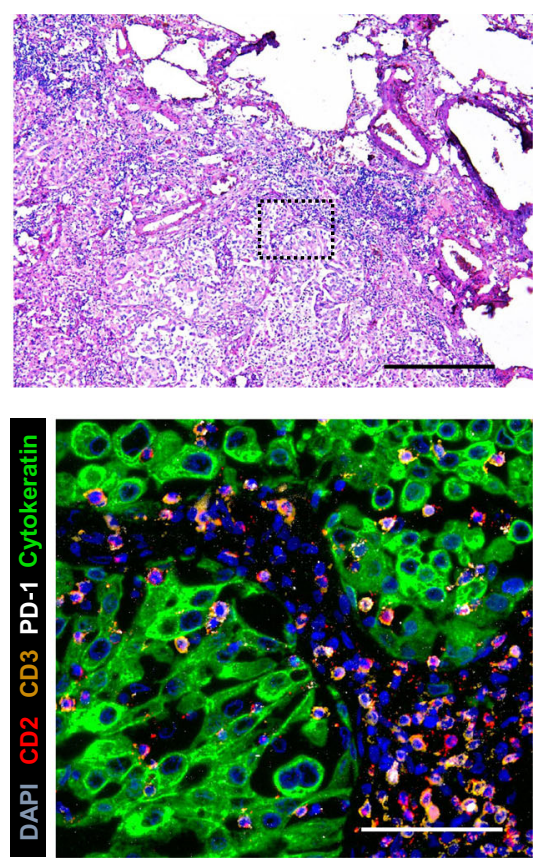

e

f

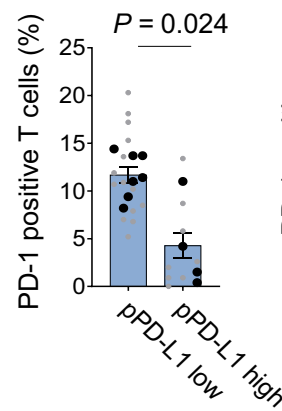

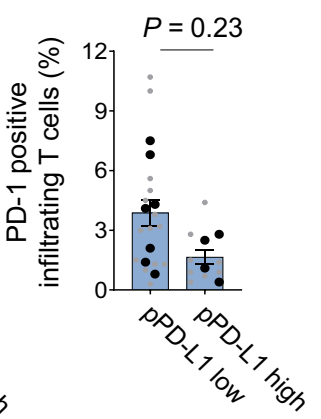

Fig. 5 pPD-L1 correlates with T cell infiltration in NSCLC. a, b Upper, Representative micrographs of NSCLC adenocarcinoma (H\&E) ( $n=11$ ). Scale bar $500 \mu \mathrm{m}$. Lower, Immunofluorescence staining for T-cells (CD2: red, CD3: orange and PD-1: white) in the TME of a NSCLC patient presenting with low pPDL1 (a) and high PPD-L1 (b). Each image is representative for at least two regions of interest (ROI) in each tumor sample. ROI were selected based on manual prestaining of DAPI. Scale bar $100 \mu \mathrm{m}(n=11)$. c-f Quantification of T cells per FoV (c), PD-1+ T cells per FoV (\%) (d), infiltrating T cells per FoV (e) and PD-1+ infiltrating T cells per FoV (\%) (f). pPD-L1 high vs. low was defined according to the median expression in this cohort. A total number of $n=22$ ROls (small symbols) were analyzed out of a total of $n=11$ patients (large symbols). Data are mean \pm SEM. Statistical significance was calculated by two-tailed Student's $t$ test $(\mathbf{d}-\mathbf{f})$ or two-tailed Mann-Whitney test $(\mathbf{c})$. Source data are provided as a Source Data file. 
a

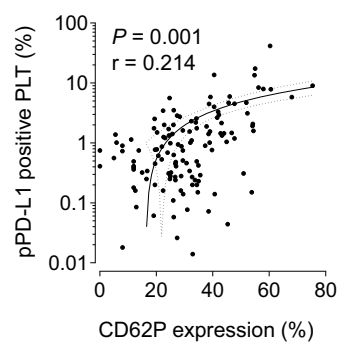

C

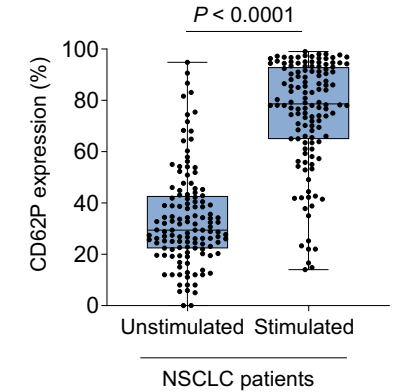

e

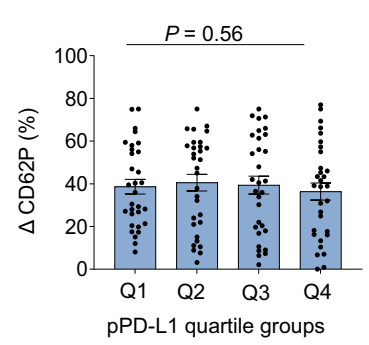

b

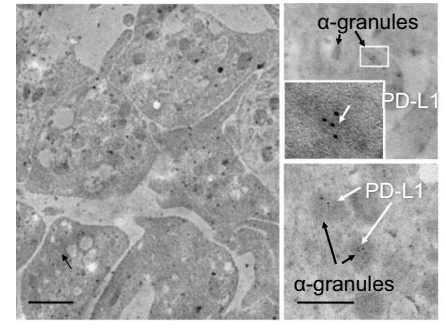

d

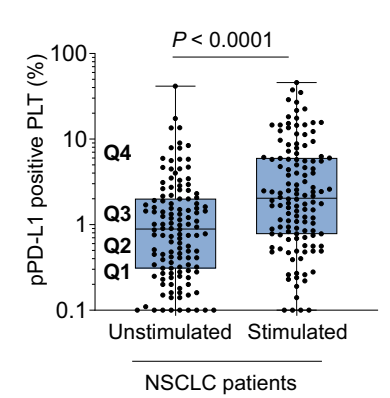

f

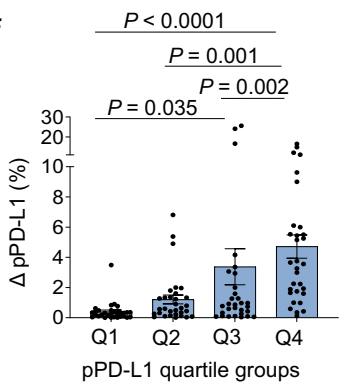

g

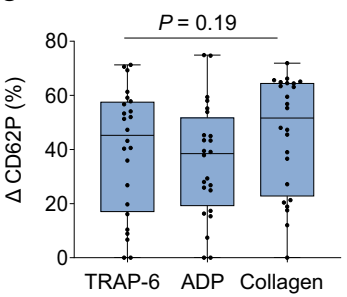

h

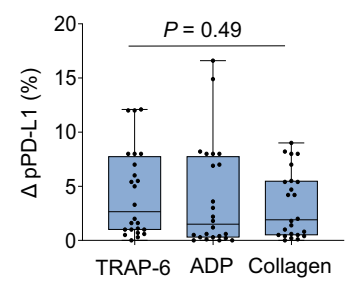

i
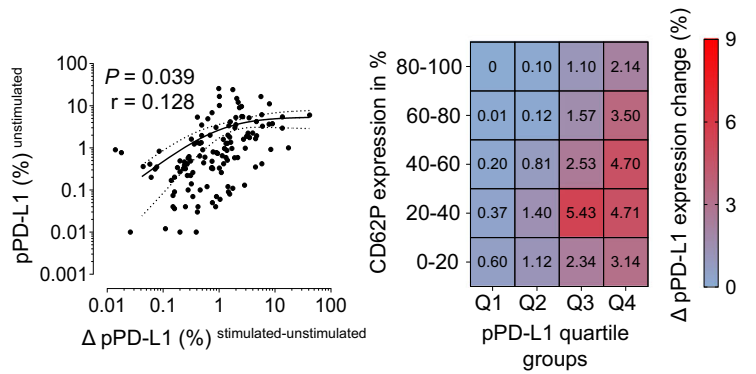

k

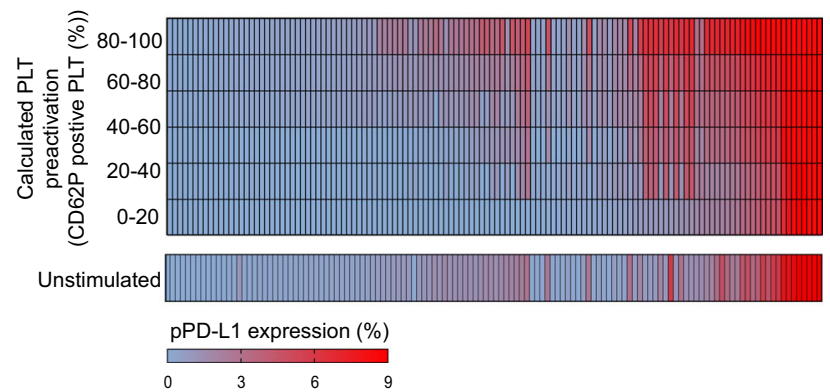

Fig. 6 Platelets from NSCLC patients show increased PD-L1 protein levels upon activation. a Correlation between platelet-derived PD-L1 (pPD-L1) and platelet activation (CD62P expression) in 128 NSCLC patients. Each dot represents a single patient. b Platelets from a NSCLC patient assessed by transmission electron microscopy. PD-L1 stained with post-embedding immunogold-labeling. Upper and lower right, PD-L1 gold particles densely accumulate in $\alpha$-granules. Scale bar left $1 \mu \mathrm{m}$, right $100 \mathrm{~nm}(n=1)$. c, d Changes in CD62P (c) and the pPD-L1 (d) levels upon platelet stimulation with $10 \mu$ M TRAP-6 for 2 min in NSCLC patient samples $(n=128)$. Boxes represent median and 25th to 75 th percentiles, whiskers are minimum to maximum. Statistical significance was calculated by two-tailed Mann-Whitney test. e CD62 expression change ( $\Delta$ CD62P) in the different pPD-L1 quartile groups identified in unstimulated platelets of NSCLC patients $(n=128)$. Data are mean \pm SEM. Statistical significance was calculated using Kruskal-Wallis test. $\mathbf{f}$ pPD-L1 expression change ( $\triangle$ pPD-L1) in the different pPD-L1 quartile groups identified in unstimulated platelets of NSCLC patients ( $n=128)$. Data are mean \pm SEM. Statistical significance was calculated by Friedman and Dunn's multiple comparisons test. $\mathbf{g}$ CD62P expression change $(\Delta C D 62 P, C D 62 P$ in stimulated platelets in \% - CD62P in unstimulated platelets in \%) after platelet activation with $10 \mu \mathrm{M}$ TRAP-6 $(n=24), 2.5 \mu \mathrm{M}$ ADP ( $n=24)$, or $5 \mu \mathrm{g} / \mathrm{ml}$ collagen $(n=24)$. Boxes represent median and 25th to 75th percentiles, whiskers are minimum to maximum. h pPD-L1 expression change $\Delta$ PPD-L1 (pPDL1 in stimulated platelets in \% - pPD-L1 in unstimulated platelets in \%) in NSCLC patients after platelet activation. Statistical significance was calculated by Kruskal-Wallis test. Boxes represent median and 25th to 75th percentiles, whiskers are minimum to maximum. i Correlation between pPD-L1 expression in unstimulated platelets of 128 NSCLC patients and the $\triangle$ PPD-L1 upon platelet stimulation with $10 \mu \mathrm{M}$ TRAP-6. Each dot represents a single patient. $\mathbf{j}$ Heatmap showing the calculated $\triangle$ PPD-L1 depending on the CD62P activation ranges ( $y$-axis) and the quartiles of pPD-L1 level ( $x$-axis) calculated in pooled data from 128 NSCLC patients. For details of subsampling and calculation used, see Methods and Supplementary Fig. 4. k Adjusted pPD-L1 levels in all 128 NSCLC patients upon calculated platelet pre-activation ranges (by CD62P expression level). a, i Correlation was determined by simple linear regression analysis. Source data are provided as a Source Data file.

anti-PD-L1 mAbs to PD-L1 expressing platelets. However, this complicates an exact determination of $\mathrm{PPD}-\mathrm{L} 1$ in these patients. Even if we did not observe a significant correlation of pPD-L1 and genetic alterations beyond KRAS, this exploratory study cohort of consecutively analyzed NSCLC patients might not be ideal to study the predictive role of pPD-L1 in NSCLC patients harboring genomic alterations including EGFR, ALK and ROS1.

Nevertheless, besides the tremendous potential of pPD-L1 Adj. as a biomarker, we believe that platelet $\mathrm{PD}-\mathrm{L} 1$ might also represent a potential target for therapeutic intervention. This presumption is supported by our observation that pPD-L1 in NSCLC patients correlates with the number of $\mathrm{T}$ cells in TME and the number of infiltrating $\mathrm{T}$ cells. Similar observations in a mouse model support this finding ${ }^{35}$. Along these lines it is tempting to speculate that $\mathrm{pPD}-\mathrm{L} 1 \mathrm{might}$ be involved in formation of the premetastatic niche by generating an immunotolerant environment at sites distant from the primary tumor (Supplementary Figs. 1, 2). Inhibition of pPD-L1 could prevent the formation of metastasis and such a concept would warrant the investigation of immune-checkpoint blocking antibodies in order 

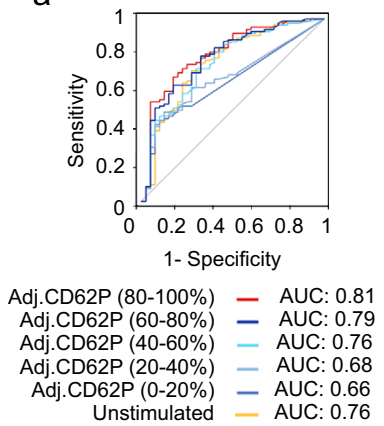

d

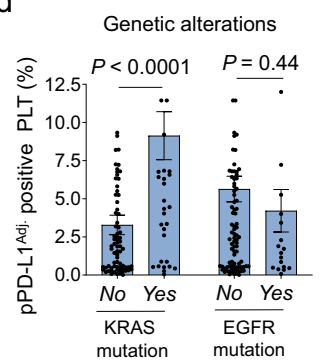

e Genetic alterations

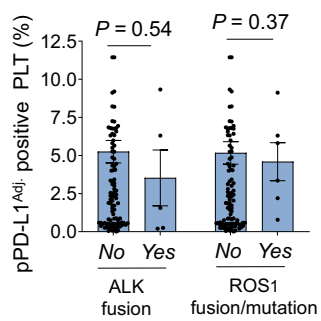

m

Platinum-based chemotherapy

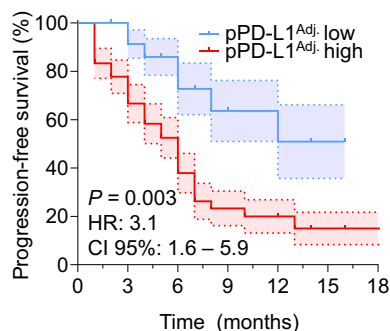

b

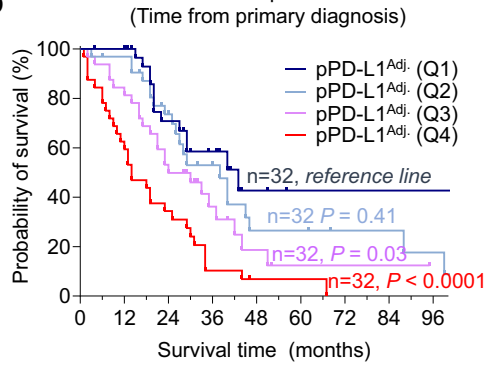

C

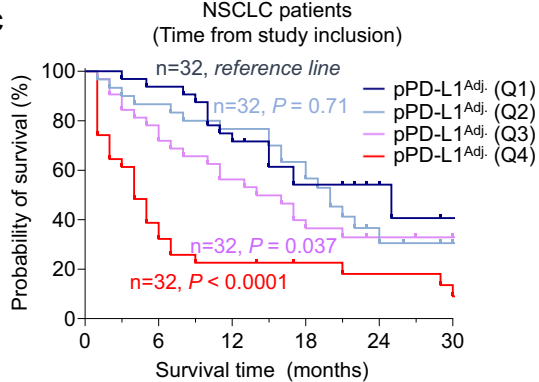

f

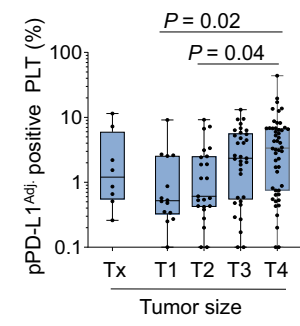

i

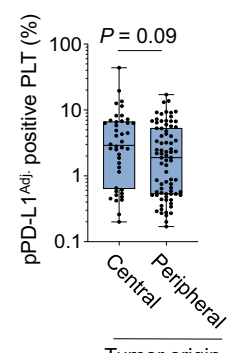

n

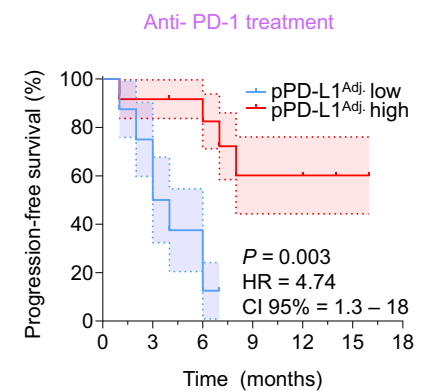

g

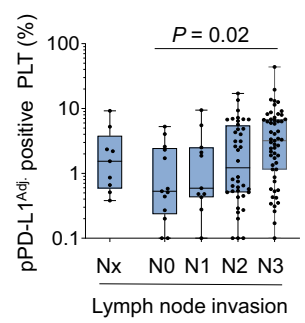

$\mathrm{k}$ h

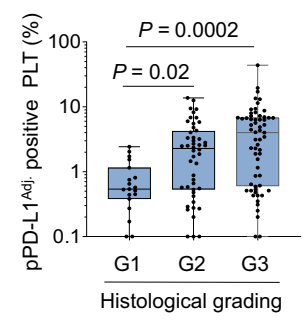

I
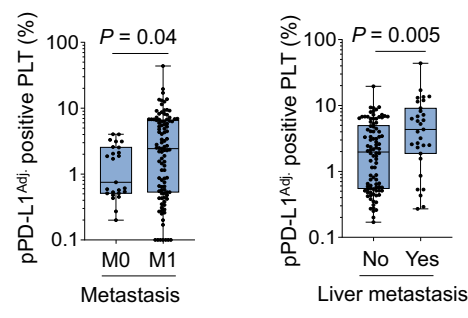

0

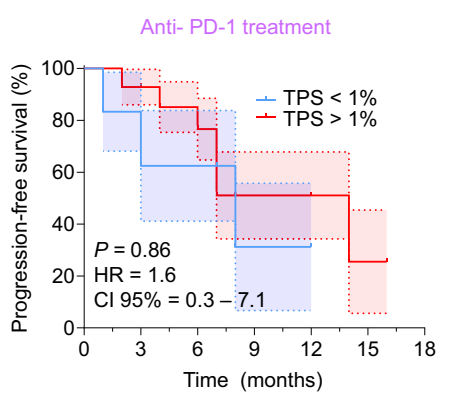

Fig. 7 Platelet-derived PD-L1 (pPD-L1) as prognostic and predictive marker in NSCLC. a Combined estimate of platelet CD62P (0-100\%) and pPD-L1 levels predicts overall survival (OS) in 128 NSCLC patients; receiver-operating characteristics (ROC) analysis. b Kaplan-Meier analysis of overall survival (OS) dependent on the pPD-L1Adj as defined by quartile groups (very low (Q1), low (Q2), high (Q3) and very high (Q4)). Survival data refer to the time point of primary diagnosis $(n=128)$. c Overall survival $(O S)$ in different pPD-L1Adj quartile groups according to the time point of platelet analysis $(n=128)$. b, c Statistical significance was calculated by log-rank test. d, e Association of pPD-L1Adj levels and different genetic alterations (KRAS (mut $=38$, wt $=$ 90), EGFR (mut =19, wt =109), EML-4-ALK (alteration =5, no alteration $=123$ ) and ROS-1 (alteration =6, no alteration 122)) (wt = wildtype, mut $=$ mutation). Data are mean \pm SEM. Statistical significance was calculated by two-tailed Mann-Whitney test. f-h pPD-L1Adj levels in patients with different tumor stage $((\mathrm{f}), \mathrm{Tx}=8, \mathrm{~T} 1=14, \mathrm{~T} 2=23, \mathrm{T3}=33, \mathrm{~T} 4=50)$, lymph node invasion $((\mathbf{g}), \mathrm{Nx}=9, \mathrm{~N} 0=13, \mathrm{~N} 1=11, \mathrm{~N} 2=40, \mathrm{~N} 3=55)$, and grade $((\mathbf{h})$, $\mathrm{G} 1=19, \mathrm{G} 2=48, \mathrm{G} 3=61$ ). Each dot represents a single patient. Statistical significance was calculated by Kruskal-Wallis test and Dunn's multiple comparisons test. $\mathbf{i}$ Association of pPD-L1Adj levels with tumor origin (central $=42$, peripheral $=79$ ). $\mathbf{j}-\mathbf{I}$ pPD-L1 Adj is associated with the occurrence of metastasis (in general $((\mathbf{j}), M 0=25, M 1=103$ ) and at specific sites, including liver (confirmed =31, no metastasis $=94$ ) and brain (confirmed $=37$, no metastasis = 91). f-I Each dot represents a single patient. Boxes represent median and 25th to 75th percentiles, whiskers are minimum to maximum. Statistical significance was calculated by two-tailed Mann-Whitney test. $\mathbf{m}$ Kaplan-Meier curves estimates of PFS in patients with a pPD-L1Adj level > median (red) and pPD-L1Adj level < median (blue) treated with conventional chemotherapy $(n=62)$. $\mathbf{n}$ Kaplan-Meier curves estimates of PFS in patients with a pPD-L1Adj level > median (red) and PPD-L1Adj level < median (blue) treated with anti-PD-1 therapy $(n=20)$. o Kaplan-Meier curves estimates of PFS in patients with a TPS score $>1 \%$ (red) and TPS $<1 \%$ (blue) treated with anti-PD- 1 therapy $(n=20)$. m-o Statistical significance was calculated by log-rank test. Source data are provided as a Source Data file. 
to prevent metastasis when tumors with high metastatic risk are treated in a curative intention. Of note, clinical trials investigating the perioperative administration of ICI in NSCLC have reported reduced relapse and metastasis and our herein presented data might offer a mechanistic explanation for the observed results $^{44,45}$. Last but not least, as pPD-L1 Adj. is shown to be prognostic and predictive in NSCLC, pPD-L1 might additionally serve as a liquid biomarker for early tumor detection or recurrence, an approach which warrants future clinical testing.

\section{Methods}

Study design and selection of patients. During 2016-2019, 173 consecutive patients with non-small lung cancer (NSCLC) treated in the Department of Medical Oncology and Hematology and Department of Internal Medicine VIII, University Hospital Tuebingen, Germany were prospectively included in the study (screening cohort $=\mathrm{SC}$ ). In order to preclude the influence of anticoagulants like aspirin (ASS), low molecular weight heparin (LMWH) or other heparinoids and non-vitamin $\mathrm{K}$ antagonist oral anticoagulants (NOACs), long-term medication of each patient was considered. In our cohort 12 patients with LMWH and 28 patients taking ASS and/or clopidogrel were excluded. In Supplementary Fig. 4, a detailed flowchart of patient selection is given. In all cases, sample collection was performed prior to the next application of the respective therapy. Tumor characteristics are based on baseline clinical staging. In order to take disease progression better into account the occurrence of metastasis was double checked at the time point of study inclusion. Our cohort comprised 71 male and 57 female patients with a mean age of 65.7 years (range 19-87). The diagnosis of a NSCLC was histologically confirmed in all cases. NSCLC adenocarcinoma was identified in 93 patients $(72.7 \%)$, in 35 cases $(27.3 \%)$ a squamous cell carcinoma was found. The details of the all patients' characteristics are summarized in Supplementary Table 1. Written informed consent was given in all cases. Sample collection of healthy participants were in accordance with the ethical standards of the institutional research committee (Ethic committee of the Faculty of Medicine of the Eberhard Karls University Tuebingen and of the University Hospital Tuebingen vote $13 / 2007 \mathrm{~V}$ ). The observational study in NSCLC patients was approved by the ethics committee of the Faculty of Medicine of the Eberhard Karls University Tuebingen and of the University Hospital Tuebingen and was conducted in accordance with the Declaration of Helsinki (vote 456/BO2).

Preparation of platelets. Platelets were obtained from healthy donors (not taking any medication for at least 10 days) and NSCLC patients after informed writing consent. Citrated blood was briefly centrifuged for $20 \mathrm{~min}$ at $120 \times \mathrm{g}$, the upper fraction was harvested as platelet-rich plasma (PRP). Platelets were washed twice with citrate wash buffer $(128 \mathrm{mmol} / \mathrm{L} \mathrm{NaCl}, 11 \mathrm{mmol} / \mathrm{L}$ glucose, $7.5 \mathrm{mmol} / \mathrm{L}$ $\mathrm{Na}_{2} \mathrm{HPO}_{4}, 4.8 \mathrm{mmol} / \mathrm{L}$ sodium citrate, $4.3 \mathrm{mmol} / \mathrm{L} \mathrm{NaH} \mathrm{PO}_{4}, 2.4$ citric acid, $0.35 \%$ bovine serum albumin, and $50 \mathrm{ng} / \mathrm{mL}$ prostaglandin $\mathrm{E}_{1}$ (PGE1)). To avoid the influence of PGE1 on platelet-tumor cell and platelet-immune cell interaction, we did not use PGE1 in our co-incubation experiments. For platelet activation $10 \mu \mathrm{M}$ of the Thrombin Receptor Activator Peptide 6 (TRAP-6), a protease-activated receptor $1\left(\mathrm{PAR}_{1}\right)$ agonist, $2.5 \mu \mathrm{M} \mathrm{ADP}$ or $5 \mu \mathrm{g} / \mathrm{mL}$ Collagen was added to the platelets for $2 \mathrm{~min}$. Platelets were fixed by $2 \%$ paraformaldehyde for $10 \mathrm{~min}$ and washed twice with PBS containing 1\% FCS.

Flow cytometry. Flow cytometry was performed using fluorescence-conjugates or specific $\mathrm{mAb}$ and their controls followed by species-specific conjugate (Supplementary Table 2) using a FACS CantoII flow cytometer (Beckman Coulter) or a LSRFortessa (Becton Dickinson) from the flow cytometer facility Tuebingen. Positive cells in percentage (\%) were calculated as follows: Surface expression in percent obtained with the specific antibody-surface expression in percent obtained with isotype control. Platelets were preselected by CD41a ${ }^{+}$and CD62 $\mathrm{P}^{-}$ (resting) or $\mathrm{CD}_{2} \mathrm{P}^{+}$(activated). Data analysis was performed using FlowJo software (v.10). In order to verify the reproducibility of our flow cytometry system, we performed a Bland-Altman analysis (Supplementary Fig. 8f). For immunophenotyping of PBMC subsets of lung cancer patients and healthy control donors were identified by counterstaining with CD3-PECy5 (BD biosciences, San Diego, CA), CD19-APC/Fire750, CD4-Pacific Blue, CD8a-BV605, CD56-PECy7, CD14-BV785, HLA-DR-BV650 (Biolegend, San Diego, CA) and CD16-FITC (invitrogen). PD-1 and PDL-1 expression as well as activation levels were analyzed using a PD-1-APC or PDL-1-APC and a CD69-PE antibody (BD biosciences), respectively. Isotype controls were obtained from BD biosciences. Dead cells were excluded from analysis with LIVE/DEAD ${ }^{\text {mi }}$ Fixable Aqua (Thermo Fisher Scientific, Waltham, MA).

\section{Histopathology, immunohistochemistry and immunofluorescence staining of} paraffin-embedded tissue samples. Tissue samples were fixed in $4 \%$ formalin and paraffin-embedded (FFPE) at the Department of Pathology (University Hospital Tuebingen). The sections were cut briefly in $3 \mu \mathrm{m}$ sections and stained with Hematoxylin/Eosin and CD61 (clone: 2C9.G3) following standard protocols. For immunofluorescence microscopy, sections were deparaffinized and hydrated in a first step. The heat-induced antigen retrieval method was performed using sodium citrate buffer ( $\mathrm{pH}$ 6.0) for $30 \mathrm{~min}$. Antigen blocking was performed with Blocking solution (Zytomed) for $60 \mathrm{~min}$. Primary antibodies that were included anti-CD41, mouse, 1:250 (clone: HIP8) and anti-PD-L1, rabbit, 1:200 (clone:28-8). Secondary antibodies include Alexa-Fluor 594 labeled anti-rabbit (1:1000, Invitrogen) and Fluor 488 labeled anti-mouse (1:1000, Invitrogen). DAPI (1:1000, BioLegend) was used for nuclear staining prior mounting the slides with $\mathrm{H}-1500$ Vectashield Hardset. Microscopic analysis was done with an Olympus BX63 microscope and a DP80 camera (Olympus).

Immunofluorescence staining of platelets and tumor cells. For immunostaining tumor cells and/or platelets were fixed in 2\% PFA in PBS ( $\mathrm{pH} 7.4$ ) for $10 \mathrm{~min}$ at $-20^{\circ} \mathrm{C}$. After three washing steps in PBS cells were incubated with a BSA blocking solution (2\% BSA, 0,2\% Triton X-100, 0,1\% Tween) for 1 hour. Primary antibodies were anti-PD-L1, rabbit (1:250, clone: $28-8)$, anti-CD41, mouse (1:400, clone: HIP8), anti-CD61, rabbit (1:250, clone: SJ-19-09), anti-GFP, rabbit (1:200, clone: EPR14104), anti-fibronectin, mouse (1:200, clone: P1H11); as secondary antibodies Alexa-Fluor 488/594 labeled anti-rabbit (1:1000, Invitrogen) and Fluor 488/594 labeled anti-mouse (1:1000, Invitrogen) were used. Afterwards slides were mounted in fluorescent mounting medium containing DAPI (1:1000, BioLegend) counter stain. For the plasma membrane staining CellMask ${ }^{\mathrm{TM}}$ (ThermoFisher) and Dil (ThermoFisher) were used according to manufactures' instructions. For nuclear staining NucBlue ${ }^{\mathrm{TM}}$ (ThermoFisher) was used. Image acquisition was performed using an Olympus BX63 microscope and a DP80 camera (Olympus) and CellSens Dimension 1.17 software. Quantification of platelets, fibronectin, and tumor cells were performed via counting fluorescence positive signals using an ImageJ script (v.1.51n and v.1.52).

Cyclic immunofluorescence staining of NSCLC patient samples. Paraffinembedded patient samples were cut in $2-5 \mu \mathrm{m}$ slices and collected on object slides. Subsequently, sections were subjected to deparaffinization and rehydration. Slides were treated with xylene for $10 \mathrm{~min}$, followed by rehydration using an ethanol dilution series of $100 \%, 95 \%, 70 \%, 50 \%$ for $5 \mathrm{~min}$ each. One last change was performed using deionized water. Heat-induced antigen retrieval was performed using a Sodium-Citrate buffer ( $10 \mathrm{mM}$ Sodium citrate, $0.05 \%$ Tween $20, \mathrm{pH} 6.0)$ and boiling the samples for $20 \mathrm{~min}$. Samples were cooled down and stored in MACSima $^{\text {m }}$ Running Buffer (Miltenyi Biotec, 130-121-565) until initial DAPI staining (Miltenyi Biotec, 130-111-570). The MACSimaTM device is an ultra-high content cyclic IF device which allows for fully automated IF imaging. Iteratively, the device performs fluorescent staining with multiple labeled antibodies, image acquisition, and bleaching per cycle. Images were generated according to the manufacturer's instructions and analyzed with the Qi Tissue Image Analysis Software. For quantification at least two ROI were selected based on manual prestaining of DAPI.

Tracking platelet-tumor cell interaction using live-cell imaging. For live-cell imaging analysis A549 cells (cultured as stated above) were used. Tumor cells were co-incubated with platelets at a platelet-tumor cell ratio of 1:1000. Platelets were added to the tumor cells directly prior image acquisition. Platelet-tumor cell interactions were analyzed using phase-contrast live-cell microscopy with frame intervals of $30 \mathrm{~s}$ for up to $40 \mathrm{~min}$ (Leica Microsystems, Thunder Imager 3D Assay; HC PL APO $40 \times / 0.95)$ using adaptive focus control. Cell positions were assigned by their center-of-mass coordinates.

Electron microscopy and immunoelectron microscopy. For transmission electron microscopy, platelets from one representative pPD-L1 high expressing NSCLC patient were used. Platelets were centrifuged and the resulting pellets were fixed for $24 \mathrm{~h}$ in Karnovsky's fixative. As previously described, Ultrathin sections were examined with a LIBRA 120 (Zeiss) operating at $120 \mathrm{kV}^{46}$. For immunoelectron microscopy, platelets were fixed and embedded in Lowicryl K4M (Polysciences) ${ }^{47}$ Samples were stained with anti-PD-L1 antibody (Abcam) and examined using a LIBRA 120 transmission electron microscope (Zeiss) at $120 \mathrm{kV}$.

ELISA. Protein levels of PD-L1 were measured using a human PD-L1 ELISA kit (Abcam, clone: 28-8) according to the recommendations of the manufacturer. All concentrations are expressed as means \pm SEM of triplicates.

Western blot. Whole-cell extracts were prepared using RIPA buffer and protein concentration was analyzed using the BioRad Dc assay. 25-50 $\mu$ g of protein were transferred to $10-15 \%$ SDS-Page and blotted on a PVDF membrane (Millipore) with a wet blot system. The membrane was blocked for $1 \mathrm{~h}$ at room temperature with Roti-Block, followed by overnight incubation with the following antibodies: anti-PD-L1, rabbit (1:2000, clone: $28-8)$, anti-fibronectin, mouse (1:250, clone: $\mathrm{P} 1 \mathrm{H} 11)$, anti-Vinculin, mouse $(1: 10,000$, clone: hVIN-1), anti- $\alpha$ tubulin $(1: 10,000$, clone $11 \mathrm{H} 10)$ and anti- $\beta$ Actin $(1: 10,000$, clone AC-15). Blots were visualized using ECL reagents (GE Healthcare) or the Super Signal West Kit (Thermo Scientific) and the ChemiDocTM MP Imaging System using the ImageLab v5.2.1 software. 
Real-time PCR. To determine mRNA abundance in several tumor cell lines we extracted mRNA in TriFast (Peqlab) according to the manufacturer's instructions. After DNAse digestion reverse transcription of total RNA was performed using random hexamers (Roche Diagnostics) and SuperScriptII reverse transcriptase (Invitrogen). Amplification of the respective genes by real-time polymerase chain reaction (RT-PCR) was performed in a total volume of $20 \mu \mathrm{l}$ using $40 \mathrm{ng}$ of cDNA, $500 \mathrm{nM}$ forward and reverse primer and 2x GoTaq ${ }^{\circledast}$ qPCR Master Mix (Promega) according to the manufacturer's protocol. Cycling conditions were as follows: initial denaturation at $95^{\circ} \mathrm{C}$ for 2 min, followed by 40 cycles of $95^{\circ} \mathrm{C}$ for $15 \mathrm{~s}, 55^{\circ} \mathrm{C}$ for $15 \mathrm{~s}$ and $68^{\circ} \mathrm{C}$ for $20 \mathrm{~s}$. For amplification the following primers were used $\left(5^{\prime}\right.$ $>3^{\prime}$ orientation): Fibronectin (FN1), fw ACCGTGGGCAACTCTGTCAA, rev CCCACTCATCTCCAACGGCA; Tissue factor (F3), fw GGCACGGGTCTTCTCC TACC, rev TGTCCGAGGTTTGTCTCCAGG; Von Willebrand Factor (VWF), fw CCTGCACCGACATGGAGGAT, rev CGTAAGTGAAGCCCGACCGA; Fibrinogen A (FBG), fw TGAAACGACTGGAGGTGGACA, rev CACGAGCTAAAGCC CTACTGC; GAPDH (GAPDH), fw TCGACAGTCAGCCGCATCTT, rev GCCC AATACGACCAAATCCGT. Real-time PCR amplifications were performed on a CFX96 Real-Time System (Biorad). All experiments were performed in duplicates and analyzed via the 7500 Software v2.0.6. The housekeeping gene GAPDH was used to standardize the amount of sample RNA.

In vitro platelet-tumor cell co-incubation and platelet adhesion. Tumor cells were coated with platelets as described previously with slight modifications ${ }^{41,48}$. Briefly summarized, PRP was obtained from fresh whole blood by centrifugation for $20 \mathrm{~min}$ at $120 \mathrm{~g}$. Platelets were washed twice with citrate wash buffer ( $128 \mathrm{mmol} / \mathrm{L} \mathrm{NaCl}, 11 \mathrm{mmol} / \mathrm{L}$ glucose, $7.5 \mathrm{mmol} / \mathrm{L} \mathrm{Na}_{2} \mathrm{HPO}_{4}, 4.8 \mathrm{mmol} / \mathrm{L}$ sodium citrate, $4.3 \mathrm{mmol} / \mathrm{L} \mathrm{NaH} \mathrm{PO}_{4}, 2.4$ citric acid, $0.35 \%$ bovine serum albumin). In some experiments platelets were pre-treated with $5 \mu \mathrm{g} / \mathrm{mL}$ anti-CD42b (clone: AK2), $20 \mu \mathrm{g} / \mathrm{mL}$ anti-integrin $ß 1$ (clone: P4C10) and anti-Integrin $\alpha 5$ (clone: JBS5) or corresponding control IgG $(20 \mu \mathrm{g} / \mathrm{mL})$ for $30 \mathrm{~min}$ at $37^{\circ} \mathrm{C}$ and $7 \% \mathrm{CO}_{2}$. Tumor cells were incubated in platelets at a platelet-tumor cell ratio of 1:1000 for $30 \mathrm{~min}$ at $37^{\circ} \mathrm{C}$ and $7 \% \mathrm{CO}_{2}$. For immunofluorescence microscopy and FACS analysis cells were fixed in $2 \%$ PFA in PBS (pH 7.4) for $10 \mathrm{~min}$ at $-20^{\circ} \mathrm{C}$ prior staining.

\section{Preparation of fibronectin matrices and platelet blocking. To prepare fibro-} nectin matrices, plates were coated with a human plasma fibronectin purified protein (R\&D, Minneapolis, MN, USA) (concentration $50 \mu \mathrm{g} / \mathrm{cm}^{2}$ ) for $120 \mathrm{~min}$. For blocking of GPIb-IX-V complex, $\alpha 5 \beta 1$ or GPIIbIIIa, washed platelets $\left(8 \times 10^{7} / \mathrm{mL}\right)$ were pre-treated with $5 \mu \mathrm{g} / \mathrm{mL}$ anti-CD42b (clone: AK2), $20 \mu \mathrm{g} / \mathrm{mL}$ anti-integrin $ß 1$ (clone: P4C10) and anti-Integrin a5 (clone: JBS5), $1 \mu \mathrm{g} / \mathrm{mL}$ Tirofiban or corresponding control $\mathrm{IgG}_{1}(20 \mu \mathrm{g} / \mathrm{mL})$ for $30 \mathrm{~min}$ at $37^{\circ} \mathrm{C}$ and $7 \% \mathrm{CO}_{2}$. After coincubation with platelets $\left(8 \times 10^{7} / \mathrm{mL}\right)$ for $30 \mathrm{~min}$ at $37^{\circ} \mathrm{C}$ and $7 \% \mathrm{CO}_{2}$, nonadherent platelets were removed via three washing steps using PBS. After removal of non-adherent platelets cells were fixed in 2\% PFA in PBS ( $\mathrm{pH}$ 7.4) for $10 \mathrm{~min}$ at $-20^{\circ} \mathrm{C}$. Platelet adhesion to fibronectin fibrils was evaluated by calculating surface coverage area and platelet count/FoV and from microscopic images using an ImageJ script (v.1.52).

\section{Plasmid construction, transfection and knockdown of NSCLC cells. For over-} expression of PD-L1 (CD274) a True-ORF-GFP-tagged expression vector was used (OriGene, RG213071, Rockville, MD, USA). Control cells were transfected using a FLAG tag. The FLAG cDNA was generated by PCR and cloned into the PD-L1GFP vector using AsiSI and MluI restriction sites. Data analysis was done using ApEv.2.0.51. Tumor cells were transfected with $2.5 \mu \mathrm{g}$ DNA (PD-L1-GFP, FLAGGFP) using Lipofectamine ${ }^{\mathrm{TM}} 3000$, in accordance with the manufacturer's instructions. For siRNA knockdown of Fibronectin, Lipofectamine ${ }^{\mathrm{TM}} 3000$ and $100 \mathrm{pmol}$ of human (FN1) siRNA Oligo Duplex (Locus ID 2335) (Origene, SR320193) was used. As scrambled negative control we used 100pmol universal scrambled negative control siRNA (Origene, SR30004).

\section{Generation of peripheral blood mononuclear cells (PBMC) and tumor cell} lines. Peripheral blood mononuclear cells (PBMC) from healthy donors were isolated using Ficol/Paque (Biochrom) density gradient centrifugation after informed consent. All tumor cell lines were cultured with 10\% FCS in Roswell Park Memorial Institute (RPMI) 1640 Medium at $37^{\circ} \mathrm{C}$ and $7 \% \mathrm{CO}_{2}$. Cell proliferation was quantified using a Neubauer chamber; for viability testing Trypan blue staining's was performed using a $0.4 \%$ trypan blue solution (Fluka). The tumor cell lines A549 (CRM-CCL-18), NCI-H460 (HTB-177), NCI-H23 (CRL-5800), NCIH226 (CRL-5826), NCI-H322 (CRL-5806), NCI-H522 (CRL-5810), HOP-62 and HOP-92 were obtained from the American Type Culture Collection (ATCC). Mycoplasma contamination was excluded via a PCR-based method.

IFN $\gamma$ ELISPOT assay in CD8 ${ }^{+} \mathbf{T}$ cells and platelet T-cell co-incubation. Freshly thawed (ex vivo) PBMCs from healthy donors were analyzed by enzyme-linked immunospot (ELISPOT) assay in duplicates. Interferon $\gamma$ (IFN $\gamma$ ) ELISPOT assays in our study were performed as described previously ${ }^{49}$. In brief, 96-well nitrocellulose plates (Millipore) were coated with $1 \mathrm{mg} / \mathrm{mL}$ anti-IFN $\gamma \mathrm{mAb}$ (Mabtech) and incubated overnight at $4^{\circ} \mathrm{C}$. In a next step, plates were blocked with human serum $(10 \%)$ for 2 hours at $37^{\circ} \mathrm{C}$. PBMCs $\left(2.5 \times 10^{5}\right.$ cells per well) were pulsed with an EBV/CMV epitope mix containing the frequently recognized peptides BRLF109-117 YVLDHLIVV ( $\left.\mathrm{A}^{*} 02\right)$ peptide and CMV pp65 ( $\left.\mathrm{A}^{*} 02\right)$ peptide NLVPMVATV and incubated with or without platelets (ratio 1:50) for $24 \mathrm{~h}$. Phytohemagglutinin was used as positive control. HLA-A*02 (KLFEKVKEV)- and B*07 (KPSEKIQVL)-restricted control peptides derived from benign tissues (HVexclusive HLA ligands) served as negative control. Prior co-incubation with T cells PD-L1 positive platelets from NSCLC patients were pre-treated with the anti-PDL1 mAB Atezolizumab for $30 \mathrm{~min}$ and washed twice with PBS containing $1 \%$ FCS Readout was performed according to the manufacturer's instructions. Spots were counted using an ImmunoSpot S5 analyzer (CTL).

Cytokine and cell surface marker staining. Peptide-specific T cells were further analyzed by intracellular cytokine and cell surface marker staining. PBMCs were incubated with $10 \mu \mathrm{g} \mathrm{ml}^{-1}$ of peptide, $10 \mu \mathrm{g} \mathrm{ml}^{-1}$ brefeldin A (Sigma-Aldrich) and a 1:500 dilution of GolgiStop (BD) for 12-16 h. Staining included Cytofix/Cytoperm solution (BD), anti-CD4, mouse (1:100, clone: RPA-T4), anti-CD8, mouse (1:400, clone: B9.11), anti-TNF, mouse (1:120, clone: Mab11) and anti-IFN- $\gamma$, mouse (1:200 dilution, clone: 4SB3). PMA $\left(5 \mu \mathrm{g} \mathrm{ml}^{-1}\right)$ and ionomycin $(1 \mu \mathrm{M}$, Sigma-Aldrich) served as positive control. Viable cells were determined using Aqua live/dead (1:400 dilution, Invitrogen). Samples were analyzed on a FACS Canto II cytometer (BD) and evaluated using FlowJo software v.10.0.8 (BD).

\section{Generation of NY-ESO-1-specific CD4 ${ }^{+} \mathrm{T}$ cells and platelet T-cell co-incu-} bation. The generation of NY-ESO-1-specific T cells was performed using as described previously $y^{50}$. In brief, PBMCs from a healthy donor $\left(1 \times 10^{7} / \mathrm{mL}\right)$ were stimulated using pools of NY-ESO-1 overlapping peptides $(1 \mu \mathrm{g} / \mathrm{mL})$. The NYESO-1 overlapping peptide pool of 15 amino acid length (11 amino acid overlap) was purchased via Miltenyi Biotec. The cells were cultured in RPMI 1640 containing $10 \%$ human $\mathrm{AB}$-serum and $1 \% \mathrm{~L}$-glutamin in the presence of $10 \mathrm{U} / \mathrm{mL}$ recombinant IL-2 and $10 \mathrm{ng} / \mathrm{mL}$ Il-7. Culture medium was replaced every third day. After a pre-sensitization period of 7-14 days, NY-ESO-1 specific, IFN $\gamma^{+}$ $\mathrm{T}$ cells were enriched after re-stimulation with NY-ESO-1 peptide pool for $6 \mathrm{~h}$ using CliniMACS ${ }^{\circledR}$ (Miltenyi Biotec) technique as reported previously ${ }^{51}$. After enrichment, NY-ESO-1 specific T cells were expanded for 14 days in the presence of IL-7 $(10 \mathrm{ng} / \mathrm{mL})$, IL-15 $(10 \mathrm{ng} / \mathrm{mL})$ and IL-2 $(50 \mathrm{U} / \mathrm{mL})$. T cell specificity was analyzed via intracellular IFN $\gamma$ staining as stated above. For further characterization of the T-cells the differentiation markers anti-CD45RO, mouse (1:200, clone: HI100), anti-CD62L, mouse (1:400, clone: DREG-56), anti-CD28, mouse (1:200, clone: $\mathrm{CD} 28.2)$ and anti-CD27, mouse (1:200, clone: M-T271) were co-analyzed by flow cytometry. For the platelet-T-cell co-incubation assay, NY-ESO-1 specific T cells $\left(5 \times 10^{6} / \mathrm{mL}\right)$ were cultured in TexMACS GMP Medium (Miltenyi Biotec). Six hours prior analysis $\mathrm{T}$ cells were co-incubated with platelets of NSCLC patients or healthy donors (ratio 1:200) and re-stimulated with NY-ESO-1 peptides $(1 \mu \mathrm{g} /$ $\mathrm{mL}$ ). In order to investigate the functional role of PD-L1 on platelets surfaces, PDL1 positive platelets from NSCLC patients were pre-treated with Atezolizumab $(100 \mu \mathrm{g} / \mathrm{mL})$ for $30 \mathrm{~min}$ and washed twice with PBS containing $1 \%$ FCS. As a negative control a Myelin oligodendrocyte glycoprotein (MOG) peptide mix was used $(1 \mu \mathrm{g} / \mathrm{mL})$. SEB (Toxin Technology, Sarasota, FL, USA) at $10 \mu \mathrm{g} / \mathrm{mL}$ was used as positive control. NY-ESO-1 specific T cell activity was determined by intracellular TNFa and IFN $\gamma$ quantified via flow cytometry as described above.

In situ proximity ligation assay (PLA). HOP-62 and NCI-H23 cells were grown on glass bottomed plates. After two washing steps cells were fixed in 1\% PFA in PBS (pH 7.4) for $10 \mathrm{~min}$ at $-20^{\circ} \mathrm{C}$. After three washing steps in PBS cells were incubated with a BSA blocking solution ( $5 \% \mathrm{BSA}, 0,2 \%$ Triton X-100, 0,1\% Tween) for 30 min. In situ PLA was performed using the Duolink PLA kit (Sigma-Aldrich) according to the manufacturer's instructions. In brief, after blocking cells were incubated with anti-PD-L1, rabbit (1:250, clone: 28-8) and anti-fibronectin, mouse (1:200, clone: P1H11) for $2 \mathrm{~h}$ at room temperature. After three washing steps with PBST (phosphate buffered saline, 0.1\% Tween), anti-mouse PLUS and anti-rabbit MINUS PLA probes were linked to the primary antibodies for $1 \mathrm{~h}$ at $37^{\circ} \mathrm{C}$. After three times washing steps with buffer A $(0.01 \mathrm{M}$ Tris, $0.15 \mathrm{M} \mathrm{NaCl}$, and $0.05 \%$ Tween-20), PLA probes were ligated for $60 \mathrm{~min}$ at $37^{\circ} \mathrm{C}$. After two washing steps with buffer A, amplification using Duolink In Situ Detection Reagents (Sigma) was performed at $37^{\circ} \mathrm{C}$ for $120 \mathrm{~min}$. Following amplification, cells were washed three times for 5 min with wash buffer B $(0.2 \mathrm{M}$ Tris $0.1 \mathrm{M} \mathrm{NaCl})$. Cells were then coated with Duolink Mounting Medium containing DAPI. Image acquisition was performed using an Olympus BX63 microscope and a DP80 camera (Olympus).

\section{Establishment of an activation-independent calculation matrix for platelet} PD-L1. Since platelet pre-activation levels differ due to sample collection/preparation and protein surface expression depends on the platelet activation state, accurate determination of total protein expression on platelet surfaces is challenging. As a result, the platelet pre-/activation level acts as a confounding factor and thus impairs the suitability of pPD-L1 as a promising biomarker in NSCLC. To circumvent this dilemma, we established an activation-independent calculation matrix of platelet PD-L1. The matrix is based on our cohort of 128 NSCLC patients and investigates the activation-dependent expression change of PD-L1 $(\triangle \mathrm{pPD}-\mathrm{L} 1)$ during controlled platelets stimulation ex vivo. Patients were categorized into PPD-L1 quartile groups 
(Q1: very low, Q2: low, Q3: medium, Q4: high), according to the pPD-L1 expression in unstimulated platelets. The pre-activation of platelets after sample preparation was determined via $\mathrm{CD} 26 \mathrm{P}$ expression. In a second step each quartile group was subdivided according to the respective pre-activation levels (CD62P expression: $0-20 \%, 20-40 \%, 40-60 \%, 60-80 \%$, and $80-100 \%$ ) according to the pre-activation levels. The activation-dependent expression changes of PD-L1 ( $\triangle \mathrm{pPD}-\mathrm{L} 1)$ was then calculated for each subgroup. An overview of the subsampling and calculation is given in Supplementary Fig. 4.

Statistics. Student's $t$ test, Mann-Whitney $U$ test, one-way ANOVA and Friedman's test were used for continuous variables, chi-squared test or Fisher's exact test for categorical variables. If significant differences by one-way ANOVA were found, group wise comparison was done (Tukey's multiple comparison test). If significant differences by Friedman's test were found Dunn's multiple comparisons test was used. Prior performing each statistical test we tested for normal distribution using the D'Agostino \& Pearson test. Overall survival (OS) and progression-free survival (PFS), including the median, were calculated using the Kaplan-Meier method. Hazard ratios (HRs) were determined using Cox regression analysis. OS was calculated from the date of primary diagnosis or time-point of study inclusion and stratified by the end of the study. The predictive value of platelet-derived PD-L1 as a prognostic factor was evaluated by examining the area under the receiveroperator characteristic (ROC) curve using a confidence interval of $95 \%$. All statistical tests were considered statistically significant when $P$ was below 0.05 . Statistical analysis was performed using SigmaStat, SPSSv21 and v27 and GraphPadPrism (v.8.1.0 and v.8.4.0).

Reporting summary. Further information on research design is available in the Nature Research Reporting Summary linked to this article.

\section{Data availability}

All data generated in this study are available within the Article, Supplementary Information or Source Data file. Source data are provided with this paper.

Received: 20 May 2021; Accepted: 9 November 2021;

Published online: 01 December 2021

\section{References}

1. Nishimura, H. et al. Autoimmune dilated cardiomyopathy in PD-1 receptordeficient mice. Science 291, 319-322 (2001).

2. Ueda, H. et al. Association of the T-cell regulatory gene CTLA4 with susceptibility to autoimmune disease. Nature 423, 506-511 (2003).

3. Leach, D. R., Krummel, M. F. \& Allison, J. P. Enhancement of antitumor immunity by CTLA-4 blockade. Science 271, 1734-1736 (1996).

4. Ishida, Y., Agata, Y., Shibahara, K. \& Honjo, T. Induced expression of PD-1, a novel member of the immunoglobulin gene superfamily, upon programmed cell death. EMBO J. 11, 3887-3895 (1992).

5. Hodi, F. S. et al. Improved survival with ipilimumab in patients with metastatic melanoma. N. Engl. J. Med. 363, 711-723 (2010).

6. Herbst, R. S. et al. Pembrolizumab versus docetaxel for previously treated, PDL1-positive, advanced non-small-cell lung cancer (KEYNOTE-010): a randomised controlled trial. Lancet 387, 1540-50 (2016).

7. Reck, M. et al. Pembrolizumab versus chemotherapy for PD-L1-positive nonsmall-cell lung cancer. N. Engl. J. Med. 375, 1823-33 (2016).

8. Yau, T. et al. Efficacy and safety of nivolumab plus ipilimumab in patients with advanced hepatocellular carcinoma previously treated with sorafenib: the CheckMate 040 randomized clinical trial. JAMA Oncol. 6, e204564 (2020).

9. Carbone, D. P. et al. First-line nivolumab in stage IV or recurrent non-smallcell lung cancer. N. Engl. J. Med. 376, 2415-26 (2017).

10. Bray, F. et al. Global cancer statistics 2018: GLOBOCAN estimates of incidence and mortality worldwide for 36 cancers in 185 countries. CA: Cancer J. Clin. 68, 394-424 (2018).

11. Siegel, R. L., Miller, K. D., Fuchs, H. E. \& Jemal, A. Cancer Statistics, 2021. CA: Cancer J. Clin. 71, 7-33 (2021).

12. Teng, F., Meng, X., Kong, L. \& Yu, J. Progress and challenges of predictive biomarkers of anti PD-1/PD-L1 immunotherapy: a systematic review. Cancer Lett. 414, 166-73 (2018).

13. Herbst, R. S. et al. Predictive correlates of response to the anti-PD-L1 antibody MPDL3280A in cancer patients. Nature 515, 563-567 (2014).

14. Taube, J. M. et al. Association of PD-1, PD-1 ligands, and other features of the tumor immune microenvironment with response to anti-PD-1 therapy. Clin. Cancer Res. 20, 5064-5074 (2014).

15. Champiat, S., Ferte, C., Lebel-Binay, S., Eggermont, A. \& Soria, J. C. Exomics and immunogenics: Bridging mutational load and immune checkpoints efficacy. Oncoimmunology 3, e27817 (2014).
16. Warshaw, A. L., Laster, L. \& Shulman, N. R. The stimulation by thrombin of glucose oxidation in human platelets. J. Clin. Invest. 45, 1923-1934 (1966).

17. Warshaw, A. L., Laster, L. \& Shulman, N. R. Protein synthesis by human platelets. J. Biol. Chem. 242, 2094-2097 (1967).

18. Weyrich, A. S. et al. Signal-dependent translation of a regulatory protein, Bcl 3, in activated human platelets. Proc. Natl Acad. Sci. USA 95, 5556-5561 (1998).

19. Evangelista, V. et al. De novo synthesis of cyclooxygenase- 1 counteracts the suppression of platelet thromboxane biosynthesis by aspirin. Circ. Res. 98, 593-595 (2006)

20. Schneider-Poetsch, T. et al. Inhibition of eukaryotic translation elongation by cycloheximide and lactimidomycin. Nat. Chem. Biol. 6, 209-17 (2010).

21. Cho, J. \& Mosher, D. F. Role of fibronectin assembly in platelet thrombus formation. J. Thromb. Haemost. 4, 1461-1469 (2006).

22. Zeiler, M., Moser, M. \& Mann, M. Copy number analysis of the murine platelet proteome spanning the complete abundance range. Mol. Cell Proteom. 13, 3435-3445 (2014).

23. Grassme, H., Bock, J., Kun, J. \& Gulbins, E. Clustering of CD40 ligand is required to form a functional contact with CD40. J. Biol. Chem. 277, 30289-30299 (2002)

24. Metelli, A. et al. Thrombin contributes to cancer immune evasion via proteolysis of platelet-bound GARP to activate LTGF- $\beta$. Sci Transl Med. 12, eaay4860 (2020)

25. Rachidi, S. et al. Platelets subvert $\mathrm{T}$ cell immunity against cancer via GARP TGF $\beta$ axis. Sci Immunol. 2, eaai7911 (2017).

26. Kim, S. H. et al. Expression of cancer-testis antigens MAGE-A3/6 and NYESO-1 in non-small-cell lung carcinomas and their relationship with immune cell infiltration. Lung 187, 401-411 (2009).

27. Mussbacher, M. et al. Optimized plasma preparation is essential to monitor platelet-stored molecules in humans. PLoS ONE 12, e0188921 (2017).

28. Hechler, B., Dupuis, A., Mangin, P. H. \& Gachet, C. Platelet preparation for function testing in the laboratory and clinic: Historical and practical aspects. Res. Pr. Thromb. Haemost. 3, 615-25 (2019).

29. Skoulidis, F. \& Heymach, J. V. Co-occurring genomic alterations in nonsmall-cell lung cancer biology and therapy. Nat. Rev. Cancer 19, 495-509 (2019).

30. Kortlever, R. M. et al. Myc cooperates with Ras by programming inflammation and immune suppression. Cell 171, 1301-1315.e14 (2017).

31. Liu, C. et al. The superior efficacy of anti-PD-1/PD-L1 immunotherapy in KRAS-mutant non-small cell lung cancer that correlates with an inflammatory phenotype and increased immunogenicity. Cancer Lett. 470, 95-105 (2020).

32. Rolfes, V. et al. PD-L1 is expressed on human platelets and is affected by immune checkpoint therapy. Oncotarget 9, 27460-70 (2018).

33. Eisenhauer, E. A. et al. noce response evaluation criteria in solid tumours: revised RECIST guideline (version 1.1). Eur. J. Cancer 45, 228-247 (2009)

34. Seymour, L. et al. iRECIST: guidelines for response criteria for use in trials testing immunotherapeutics. Lancet Oncol. 18, e143-e52 (2017).

35. Zaslavsky, A. B. et al. Platelet PD-L1 suppresses anti-cancer immune cell activity in PD-L1 negative tumors. Sci. Rep. 10, 19296 (2020).

36. Draga, P. E. et al. PD-L1 expression on circulating tumor cells and platelets in patients with metastatic breast cancer. PLoS ONE 16, e0260124 (2021).

37. Mammadova-Bach, E. et al. Platelet integrin a6 61 controls lung metastasis through direct binding to cancer cell-derived ADAM9. JCI Insight 1, e88245 (2016).

38. Pearlstein, E., Salk, P. L., Yogeeswaran, G. \& Karpatkin, S. Correlation between spontaneous metastatic potential, platelet-aggregating activity of cell surface extracts, and cell surface sialylation in 10 metastatic-variant derivatives of a rat renal sarcoma cell line. Proc. Natl Acad. Sci. USA 77, 4336-4339 (1980).

39. Heinmöller, E. et al. Studies on tumor-cell-induced platelet aggregation in human lung cancer cell lines. J. Cancer Res Clin. Oncol. 122, 735-744 (1996).

40. Bastida, E., Escolar, G., Almirall, L. \& Ordinas, A. Platelet activation induced by a human neuroblastoma tumor cell line is reduced by prior administration of ticlopidine. Thromb. Haemost. 55, 333-337 (1986).

41. Placke, T. et al. Platelet-derived MHC class I confers a pseudonormal phenotype to cancer cells that subverts the antitumor reactivity of natural killer immune cells. Cancer Res. 72, 440-448 (2012).

42. Blair, P. \& Flaumenhaft, R. Platelet alpha-granules: basic biology and clinical correlates. Blood Rev. 23, 177-189 (2009).

43. Zito Marino, F. et al. Heterogeneity of PD-L1 Expression in Lung Mixed Adenocarcinomas and Adenosquamous Carcinomas. Am. J. Surg. Pathol. 44, 378-86 (2020).

44. Cascone, T. W. W. et al. Neoadjuvant nivolumab (N) or nivolumab plus ipilimumab (NI) for resectable non-small cell lung cancer (NSCLC): Clinical and correlative results from the NEOSTAR study. J Clin Oncol. 37, 8504 (2019).

45. Ren, S., Wang, C., Shen, J. \& Zhu, C. Neoadjuvant immunotherapy with resectable non-small cell lung cancer: recent advances and future challenges. $J$. Thorac. Dis. 12, 1615-1620 (2020). 
46. Schroeder, B. O. et al. Reduction of disulphide bonds unmasks potent antimicrobial activity of human beta-defensin 1. Nature 469, 419-423 (2011).

47. Riess, T. et al. Bartonella adhesin a mediates a proangiogenic host cell response. J. Exp. Med. 200, 1267-1278 (2004).

48. Jurasz, P., Alonso-Escolano, D. \& Radomski, M. W. Platelet-cancer interactions: mechanisms and pharmacology of tumour cell-induced platelet aggregation. Br. J. Pharm. 143, 819-826 (2004).

49. Widenmeyer, M. et al. Promiscuous survivin peptide induces robust CD4+ T-cell responses in the majority of vaccinated cancer patients. Int J. Cancer 131, 140-149 (2012).

50. Kayser, S. et al. Rapid generation of NY-ESO-1-specific CD4(+) THELPER1 cells for adoptive T-cell therapy. Oncoimmunology 4, el002723 (2015).

51. Feuchtinger, T. et al. Clinical grade generation of hexon-specific $\mathrm{T}$ cells for adoptive T-cell transfer as a treatment of adenovirus infection after allogeneic stem cell transplantation. J. Immunother. 31, 199-206 (2008).

\section{Acknowledgements}

The authors thank E. Rist, P. Schiemann, A. Heinrich, E. Enzinger, L. Herrmann and all members of the Zender department for fruitful discussions and/or technical assistance. Flow cytometry sample acquisition was performed on shared instruments of the Flow Cytometry Core Facility Tuebingen. This work was supported by the German Research Foundation (DFG) FOR2314 (L.Z.), project number 267467939; SFB-TR209 (L.Z.) project number 314905040), Gottfried Wilhelm Leibniz Program (L.Z.), Germany's excellence strategy, EXC 2180-390900677, Image Guided and Functionally Instructed Tumor Therapies (iFIT) (L.Z.) and SFB-TR240, project B05 (L.Z., O.B.). Further funding was provided by the European Research Council (CholangioConcept (L.Z.)) and the German Center for Translational Cancer Research (DKTK, L.Z.). Cartoons in Supplementary Figures 1-2 were created with BioRender.com.

\section{Author contributions}

C.H., H.-G.K., O.B., and L.Z. conceived and designed the study. C.H., E.M., M.S., St.Ma., M.L., Me.He., So.Sc., C.S. conducted in vitro experiments. B.W. performed live-cell imaging. T.B., M. L., and J.W. conducted in vitro T cell experiments. C.H. J.S., Ma.Hi., J.H. Ma.Hśo., Sv.Ma., St.Si., and F.F. conducted patient data and sample collection as well as medical evaluation and analysis. C.H., M.L., J.H., and So.Sc. analyzed data and performed statistical analyses. C.H., B.W., So.Sc., Ma.Hi., Me.He, and T.B. prepared figures and tables; C.H. wrote the first draft of the manuscript. R.H., H.S., H.-G.K., O.B., and L.Z contributed to data interpretation and manuscript edit. L.Z., R.H., J.W., and O.B. gave technical support and conceptual advice. All authors critically reviewed, read, and approved the final manuscript.

\section{Competing interests}

The authors declare no competing interests.

\section{Additional information}

Supplementary information The online version contains supplementary material available at https://doi.org/10.1038/s41467-021-27303-7.

Correspondence and requests for materials should be addressed to Lars Zender.

Peer review information Nature Communications thanks Christophe Dubois and the other, anonymous, reviewer(s) for their contribution to the peer review of this work.

Reprints and permission information is available at http://www.nature.com/reprints

Publisher's note Springer Nature remains neutral with regard to jurisdictional claims in published maps and institutional affiliations.

(c) (i) Open Access This article is licensed under a Creative Commons Attribution 4.0 International License, which permits use, sharing, adaptation, distribution and reproduction in any medium or format, as long as you give appropriate credit to the original author(s) and the source, provide a link to the Creative Commons license, and indicate if changes were made. The images or other third party material in this article are included in the article's Creative Commons license, unless indicated otherwise in a credit line to the material. If material is not included in the article's Creative Commons license and your intended use is not permitted by statutory regulation or exceeds the permitted use, you will need to obtain permission directly from the copyright holder. To view a copy of this license, visit http://creativecommons.org/ licenses/by/4.0/.

(C) The Author(s) 2021 